\title{
A multilevel Bayesian method for ultrasound-based damage identification in composite laminates
}

\author{
Juan Chiachío ${ }^{\mathrm{a}, *}$, Nicolas Bochud ${ }^{\mathrm{a}}$, Manuel Chiachío ${ }^{\mathrm{a}}$, Sergio Cantero ${ }^{\mathrm{a}}$, Guillermo Rus ${ }^{\mathrm{a}}$ \\ ${ }^{a}$ Department of Structural Mechanics and Hydraulic Engineering, Campus de Fuentenueva s/n, 18071 Granada, Spain
}

\begin{abstract}
Estimating deterministic single-valued damage parameters when evaluating the actual health state of a material has a limited meaning if one considers not only the existence of measurement errors, but also that the model chosen to represent the damage behavior is just an idealization of reality. This paper proposes a multilevel Bayesian inverse problem framework to deal with these sources of uncertainty in the context of ultrasound-based damage identification. Although the methodology has a broad spectrum of applicability, here it is oriented to model-based damage assessment in layered composite materials using through-transmission ultrasonic measurements. The overall procedure is first validated on synthetically generated signals and then evaluated on real signals obtained from a post-impact fatigue damage experiment in a cross-ply carbon-epoxy laminate. The evidence of the hypothesized model of damage is revealed as a suitable measure of the overall ability of that candidate hypothesis to represent the actual damage state observed by the ultrasound, thus avoiding the extremes of over-fitting or under-fitting the ultrasonic signal. Keywords: Bayesian inverse problem, Ultrasound, Composite Laminates, Diagnostics
\end{abstract}

\section{Introduction}

Composites are high-performance layered materials that are increasingly used as primary material for engineering structures and mechanisms in the aerospace, wind energy and naval industries, among others [1, 2]. However, they are vulnerable to damage during operation, e.g., fatigue-induced damage or impact damage, that can be noticeable from the beginning of lifespan as an alteration of macro-scale mechanical properties like stiffness or strength $[3,4]$. Unlike metals, damage degradation in composites consists in a complex multiscale process driven by internal fracture mechanisms distributed through the thickness, such as micro-cracks, delaminations, fibers breakage, etc. [3-5]. These damages are hardly ever detectable by visual inspection and typically require advanced nondestructive evaluation (NDE) techniques.

*Corresponding author. e-mail: jchiachio@ugr.es Tel:(+34)958240037 Fax:(+34)958249959

E.T.S. de Ingenieros de Caminos, Canales y Puertos. 
Ultrasound is currently one of the most frequently used NDE inspection techniques mainly due to its efficiency in obtaining indirect measurements of the actual mechanical properties of materials at relatively low cost. In ultrasound-based NDE, the received ultrasonic signal is evaluated and processed to retrieve quantitative information about the state of health of the inspected media. However, given the complexity of the internal structure of composite laminates (e.g., heterogeneity, multiple damage mechanisms, etc.), ad hoc signal processing techniques are usually required for a more in depth interpretation of the measured ultrasonic signal $[6,7]$. The noise arising from the imperfections of both the acquisition system and the propagation path, and the difficulties in understanding and analyzing multiple and overlapping ultrasonic echoes, suggest to directly compare the experimental signal response with theoretical signals obtained from a model of ultrasound wave propagation (UWP), with the purpose of inferring quantitative information about the effective mechanical properties of the material. A suitable approach for such comparison is given by the model-based inverse problem (IP), in either its deterministic version [8, 9] or alternatively in its Bayesian (probabilistic) version [10-12]. The deterministic approach has been previously applied in the context of ultrasound-based damage identification in composites [7, 13], although it suffers from a strong practical limitation since it requires the adoption of an a priori hypothesis about the through-the-thickness distribution of the damaged layers. However, when dealing with composite materials under fatigue or impact degradation, not only one single hypothesis about damage distribution but numerous candidate hypotheses can be considered, even under nominally identical material and testing conditions [14, 15]. Moreover, reconstructing the damage state of a material using a deterministic IP approach neglects not only the uncertainty arising from measurement errors, but also the uncertainty related to the modeler's choice of a particular hypothesis of damage to solve the inverse problem $[11,16]$. In this context, it seems reasonable to explore the applicability of a probabilistic IP to ultrasound-based damage identification in composite materials, precisely where the benefits of the Bayesian approach can be fully exploited to deal with the aforementioned sources of uncertainty.

There are few articles in the literature dealing with some form of damage identification in composite laminates using Bayesian IP approaches. Gros [17] used several NDE methods (no ultrasound) to detect and size delamination in composites. A Bayesian framework was adopted to fuse various sources of experimental data and assess the probability of a defect to be detected at a particular location. Peng et al. [18] developed a Bayesian imaging method to detect and size delamination damage in composites using Lamb waves. Recently, some authors have made use of recursive Bayesian updating techniques for on-line damage identification in the context of fatigue life prediction in composite materials [19-21]. Other researchers have adopted Bayesian IP approaches for ultrasound-based feature identification in biomaterials and soft tissue [22, 23]. However, to the authors' best knowledge, the use of a full Bayesian inverse problem for damage assessment based on ultrasound in composite materials is still missing in the open literature.

In this paper, a multilevel Bayesian framework is proposed for identifying the through-the-thickness 
position and the effective mechanical properties of the damaged layers in composite laminates using ultrasound. As a key contribution, the proposed methodology does not require any predefined hypothesis on the damage distribution for solving the IP. Instead, several candidate damage hypotheses or model classes [11] are formally tested and ranked through relative probabilities within a Bayesian model selection framework. The chosen UWP model is based on a digital representation of the laminate recently developed by Bochud et al. [13], which is particularly suitable for the proposed Bayesian methodology due to its high efficiency and low computational complexity. In this context, Bayes' Theorem is applied at three hierarchical levels: first, to deal with the posterior information about the model parameters for a specific damage hypothesis; second, to assess the relative plausibility of each damage hypothesis within a set of candidate hypotheses defining a particular damage pattern, and third, to obtain the degree of plausibility of a given pattern of damage among a set of candidates. In this work, the concept of damage hypothesis is physically associated with the type of damage and its through-the-thickness distribution within the laminate, whereas damage pattern is defined as a set of damage hypotheses that share the same amount of damaged layers, regardless of their position. An algorithm is proposed to efficiently explore the set of possible damage hypotheses, thus avoiding an exhaustive search across an intractable number of combinations of model parameters and making the identification problem computationally feasible. In this sense, it constitutes a major contribution of this research.

To serve as a validation, the proposed Bayesian framework is initially applied to a set of synthetic signals with increasing levels of noise and complexity, which are intended to serve as ground-truth data. Additionally, a case study is presented using ultrasonic signals obtained from a post-impact fatigue damage experiment in a cross-ply carbon fiber-reinforced polymer (CFRP) laminate. Results show that the proposed methodology is able to detect and locate the damaged layers and estimate their effective mechanical properties through probabilities that measure and rank the extent of agreement between the measured ultrasonic signal and the modeled signal. It is also shown that more complex damage hypotheses (i.e., model parameterizations that involve more updatable model parameters) do not necessarily yield higher probabilities in explaining the observed ultrasonic signature even for severe damage scenarios. The last is an instance of the Principle of Model Parsimony or Ockham's razor [24, 25] which is shown to appear in a natural and principled way from the computation of the evidence of each damage hypothesis [11, 26]. Thus, it is a key aspect in favour of the proposed Bayesian approach over commonly used methods for hypotheses assessment like the Maximum Likelihood Estimation (MLE), or the information criteria like the Akaike's Information Criterion (AIC) [27] and the Bayesian Information Criterion (BIC) [28, 29]. The MLE approach is purely based on the goodness of the data-fit of the hypothesized model, thus favouring unnecessary complex damage hypotheses [11] (i.e., those that lead to only slightly better agreement with the data). The AIC and BIC criteria were proposed to attempt to correct for the bias of MLE by the addition of a penalty term to compensate for the over-fitting of more complex models. However, despite their simplicity, they may give 
biased identifications favouring excessively simple hypotheses $[11,26]$. In this context, the evidence of each damage hypothesis is revealed as a suitable measure of the overall ability of the candidate damage hypothesis to represent the actual damage state observed by the ultrasound, since it explicitly builds in a tradeoff between the goodness of fit of the hypothesized model and its information-theoretic complexity, thus avoiding the extremes of over-fitting or under-fitting the ultrasonic signal.

The paper is organized as follows. Section 2 presents the formulation adopted for modeling ultrasound waves in layered media. Key mathematical definitions for damage hypothesis and damage pattern are also introduced in this section. In Section 3, the proposed Bayesian framework for damage identification is presented. This section also provides a pseudocode implementation of the proposed search algorithm. Section 4 illustrates the proposed methodology using both, a set of synthetically generated signals and experimental ultrasonic signals. Section 5 discusses the results, and finally Section 6 provides concluding remarks.

\section{Physical fundamentals}

\subsection{Ultrasound wave propagation model}

For the purpose of ultrasound model-based damage identification, a model for ultrasound wave propagation (UWP) is required. In general terms, an UWP model can be defined as a deterministic relationship $\overline{\mathbf{y}}=\mathbf{g}(\mathbf{x}, \boldsymbol{\theta}): \mathbb{R}^{n_{\mathbf{x}}} \times \mathbb{R}^{n_{\boldsymbol{\theta}}} \rightarrow \mathbb{R}^{n_{\mathbf{y}}}$ between a sampled input signal $\mathbf{x}=\left(x_{1}, \ldots, x_{i}, \ldots, x_{n_{\mathbf{x}}}\right) \in \mathbb{R}^{n_{\mathbf{x}}}$ and a sampled output signal $\overline{\mathbf{y}}=\left(\bar{y}_{1}, \ldots, \bar{y}_{i}, \ldots, \bar{y}_{n_{\mathbf{y}}}\right) \in \mathbb{R}^{n_{\mathbf{y}}}$, with $\boldsymbol{\theta} \in \boldsymbol{\Theta} \subset \mathbb{R}^{n_{\boldsymbol{\theta}}}$ as model parameters. In a recent work, the authors developed a new modeling approach to efficiently describe the interactions of a normally-incident plane longitudinal ultrasonic wave with multilayered materials [13]. Basically, this modeling approach relies on a computational procedure that takes the effective mechanical properties of the $N_{L}$ layers of a laminate (i.e., Young's modulus $E_{\ell}$, Poisson ratio $\nu_{\ell}$, density $\rho_{\ell}$, attenuation coefficient $\alpha_{\ell}$, and thickness $t_{\ell}$, where $\ell=1, \ldots, N_{L}$ ) and analytically converts them to the coefficients of a functional digital filter in the $z$-domain [30], as depicted in Figure 1. This approach is adopted here, so that a composite laminate can be represented by a discrete-time transfer function $H(z ; \boldsymbol{\theta})$ that relates the sampled input signal $\mathbf{x}$ and the output signal $\overline{\mathbf{y}}$ as follows,

$$
\overline{\mathbf{y}}=H(z ; \boldsymbol{\theta}) \cdot \mathbf{x}
$$

This method has been demonstrated to provide high efficiency and low computational complexity [13], which is particularly beneficial for the Bayesian approach proposed in this paper since thousands of forward model evaluations are required for damage inference. In addition, it has been shown that under a through-transmission configuration, the discrete-time transfer function $H(z ; \boldsymbol{\theta})$ for a multilayered material 
corresponds to a delayed all-pole filter with sparse coefficients [13], so that:

$$
H(z ; \boldsymbol{\theta})=\frac{b_{0}}{1+\sum_{k=1}^{2 \Lambda} a_{k} z^{-k}} z^{-\Lambda} \quad \text { with } \quad\left\|a_{k}\right\|_{0} \ll 2 \Lambda .
$$

where $b_{0} \in \mathbb{R}^{+}$is a gain parameter obtained as a function of the transmission $G_{t_{\ell}} \in \mathbb{R}^{+}$and attenuation $G_{\alpha_{\ell}} \in \mathbb{R}^{+}$coefficients across the $N_{L}$ layers, and $\Lambda \in \mathbb{R}^{+}$corresponds to a sample delay equivalent to the time needed by the incident wave to cross the total thickness of the laminate. Henceforth, a composite laminate can be modeled as a linear time-invariant (LTI) sparse digital filter (with a rational transfer function in the $z$-domain) whose coefficients are analytically related to the effective mechanical properties of the layers of the laminate. See Appendix A for further details.

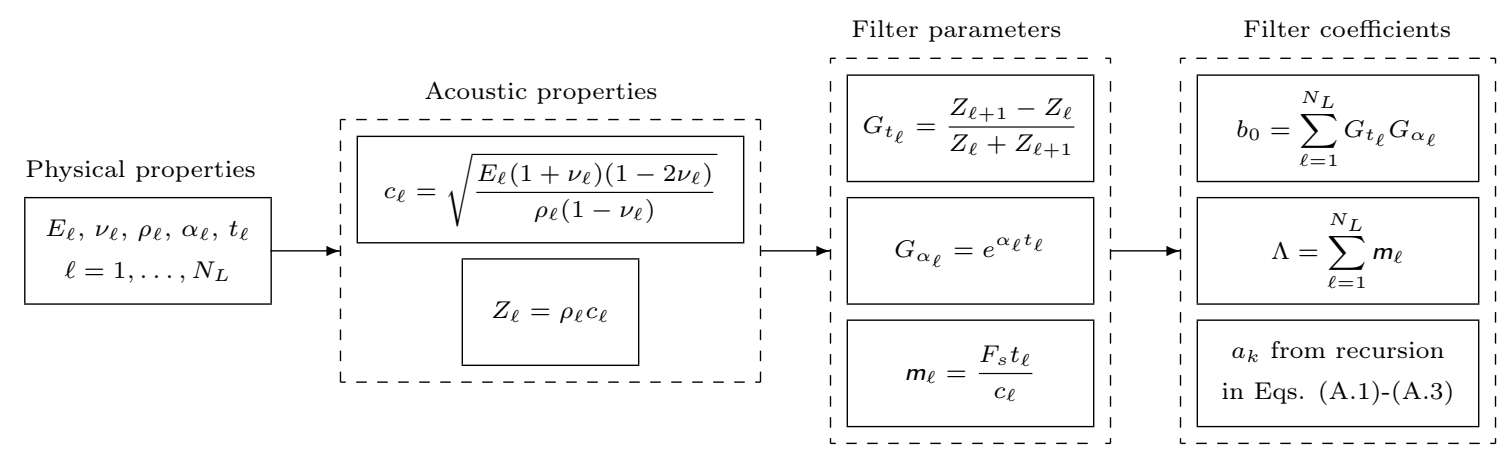

Figure 1: Computational procedure to obtain the coefficients of a functional digital filter from the basic physical properties of the laminate $\left(c_{\ell}\right.$ : wave velocity, $Z_{\ell}$ : acoustic impedance, $m_{\ell}$ : sample delay, $F_{s}$ : sampling frequency. Subscript $\ell$ refers to the $\ell$-th layer).

\subsection{Damage hypotheses/patterns definition through model parameterization}

As depicted in Figure 1, the UWP model presented above depends on a vector $\mathbf{m} \in \mathbb{R}^{n_{\mathrm{m}}}$ of parameters that describe the mechanical and geometrical properties of the layers [13], i.e., $\mathbf{m}=\left(E_{\ell}, \nu_{\ell}, \rho_{\ell}, \alpha_{\ell}, t_{\ell}\right)$, $\ell=1, \ldots, N_{L}$, where $N_{L}$ is the number of layers of the laminate. Some of these parameters are deterministic input parameters that can be represented by single-valued variables, however other parameters within $\mathbf{m}$ may be uncertain, i.e., they cannot be represented by single-valued variables but through a probability density function (PDF). These uncertain parameters are grouped into the parameter vector $\boldsymbol{\theta}$ defined over the parameter space $\Theta \subset \mathbb{R}^{n \boldsymbol{\theta}}$, that represents the region of plausible values for $\boldsymbol{\theta}$.

In this work, we assume that any damage hypothesis in a composite laminate (i.e., the expected type of damage and its through-the-thickness distribution) can be described as a uniform reduction of the Young's modulus of several layers at the inspected point or area, which is a reasonable assumption in the context of the sub-wavelength wave propagation technique adopted in this paper [31]. Consequently, the Young's moduli of the damaged layers are considered as uncertain model parameters, hereinafter called model parameters; i.e., $\boldsymbol{\theta}=\left(E_{k}, E_{l}, \ldots, E_{m}\right) \in \boldsymbol{\Theta}$, where $k, l, \ldots, m \in \Xi=\left\{1,2, \ldots, N_{L}\right\} \subset \mathbb{N}$ represent the 
through-the-thickness position of the damaged layers. As it is defined, a given parameter vector $\boldsymbol{\theta}$ implicitly carries out information about the number $\left(n_{\boldsymbol{\theta}}=\right.$ dimension of $\left.\boldsymbol{\Theta}\right)$ and position $(\{k, l, \ldots, m\} \in \Xi)$ of the damaged layers, thus defining a particular hypothesis of damage denoted by $\mathcal{M}$. By varying the position of the $n_{\boldsymbol{\theta}}$ damaged layers within the laminate, a set of $N_{M}$ candidate damage hypotheses is obtained $\left\{\mathcal{M}_{1}, \ldots, \mathcal{M}_{N_{M}}\right\}$, which defines a specific damage pattern $\mathbf{M}_{i}=\left\{\mathcal{M}_{1}, \ldots, \mathcal{M}_{N_{M}}\right\}$. Therefore, a different damage pattern $\mathbf{M}_{i^{\prime}}$ can be obtained by adopting a number $n_{\boldsymbol{\theta}}^{\prime} \neq n_{\boldsymbol{\theta}}$ of damaged layers. Observe that the damage hypotheses in the set $\mathbf{M}_{i}=\left\{\mathcal{M}_{1}, \ldots, \mathcal{M}_{N_{M}}\right\}$ share a common amount of damaged layers, namely $n_{\boldsymbol{\theta}}$, thus the notation $\mathbf{M}_{i=n_{\boldsymbol{\theta}}}$ is adopted for convenience.

\section{Bayesian methodology}

Damage hypotheses described above are just an idealization of reality, and many of them can be formulated to reproduce the experimental signal just by adopting a particular set of model parameters. To select among the many possibilities, a Bayesian framework is proposed to rank the candidate damage hypotheses and damage patterns through probabilities that measure the extent of agreement between the measured signal and the corresponding modeled signal.

\subsection{General assumptions}

For the purpose of Bayesian inference, a probability-based description of the UWP model is firstly required. Let us consider the deterministic relationship $\overline{\mathbf{y}}=\mathbf{g}(\mathbf{x}, \boldsymbol{\theta})$ between the input signal $\mathbf{x} \in \mathbb{R}^{n_{x}}$ and the output signal $\overline{\mathbf{y}} \in \mathbb{R}^{n_{y}}$ as a function of the uncertain parameters $\boldsymbol{\theta} \in \boldsymbol{\Theta} \subset \mathbb{R}^{n_{\theta}}$, as previously defined in Section 2.1. A probabilistic version of the UWP model can be defined by adding an error term $\mathbf{e} \in \mathbb{R}^{n_{y}}$, that represents the difference between the modeled signal $\overline{\mathbf{y}}$ and the experimental one $\mathbf{y}$ :

$$
\mathbf{y}=\overline{\mathbf{y}}+\mathbf{e}
$$

In the last equation, the error term $\mathbf{e}$ is assumed to be modeled as a zero-mean Gaussian distribution with covariance matrix $\boldsymbol{\Sigma}_{\mathbf{e}}$, i.e., $\mathbf{e} \sim \mathcal{N}\left(0, \boldsymbol{\Sigma}_{\mathbf{e}}\right)$. This assumption is supported by the Principle of Maximum Information Entropy (PMIE) [11, 32], which provides a rational way to establish a probability model for the error term e such that it produces the largest uncertainty (largest Shannon entropy). It follows that the probability-based version of the UWP model can be described as a Gaussian distribution:

$$
p(\mathbf{y} \mid \mathbf{x}, \boldsymbol{\theta}, \mathcal{M})=\left((2 \pi)^{n_{\mathbf{y}}}\left|\boldsymbol{\Sigma}_{\mathbf{e}}\right|\right)^{-\frac{1}{2}} \exp \left(-\frac{1}{2}(\mathbf{y}-\overline{\mathbf{y}})^{T} \boldsymbol{\Sigma}_{\mathbf{e}}^{-1}(\mathbf{y}-\overline{\mathbf{y}})\right)
$$

where the covariance operator $\boldsymbol{\Sigma}_{\mathbf{e}}$ is assumed to be a diagonal matrix, i.e. $\boldsymbol{\Sigma}_{\mathbf{e}}=\sigma_{e}^{2} \mathbf{I}_{n_{\mathbf{y}}}$, with $\mathbf{I}_{n_{\mathbf{y}}}$ being the $n_{\mathbf{y}} \times n_{\mathbf{y}}$ identity matrix and $\sigma_{e}$ the corresponding standard deviation of the error term e. The last implies that the component errors $e_{i} \in \mathbf{e}, i=1, \ldots, n_{\mathbf{y}}$, which measure the difference between the measured signal 


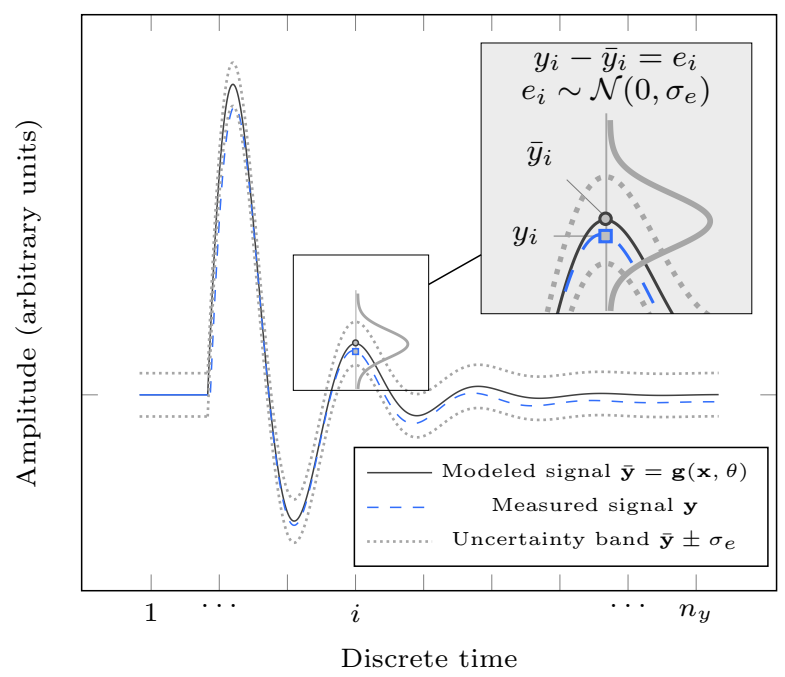

Figure 2: Illustrative example for obtaining the probability-based version of the UWP model described in Equation (5).

$\mathbf{y}$ and the modeled signal $\overline{\mathbf{y}}$, are mutually independent and identically distributed (see Figure 2 for further details). Therefore, the probabilistic UWP model in Equation (4) can be simplified as:

$$
p(\mathbf{y} \mid \mathbf{x}, \boldsymbol{\theta}, \mathcal{M})=\left(2 \pi \sigma_{e}^{2}\right)^{-\frac{n_{\mathbf{y}}}{2}} \exp \left(-\frac{1}{2} \sum_{i=1}^{n_{\mathbf{y}}}\left(\frac{y_{i}-\bar{y}_{i}}{\sigma_{e}}\right)^{2}\right)
$$

where $\mathcal{M}$ in Equations (4) and (5) denotes the candidate model class, which actually represents a particular parameterization for the UWP model, and consequently, a particular hypothesis of damage. As a part of the model class $\mathcal{M}$, we can define a prior PDF $p(\boldsymbol{\theta} \mid \mathcal{M})$ over the model parameter space $\boldsymbol{\Theta}$, which represents the initial relative plausibility of $\boldsymbol{\theta}$ before the information from measurements is incorporated, as will be explained in the next section.

\subsection{Bayesian updating}

Note that, up to this point, the probabilistic UWP model defined in Equation (5) is restricted to the adoption of a particular parameter vector $\boldsymbol{\theta}$, which automatically defines a particular damage hypothesis for the system denoted by $\mathcal{M}_{j}$. However, different values of model parameters and even different damage hypotheses might be consistent with the data $\mathcal{D}$ (i.e., experimental signal). To this end, the Cox-Jaynes theory of probability [33, 34] provides us a rigorous foundation for the Bayesian inversion, which allows obtaining the probability, in the sense of degree of plausibility, of model parameters, model hypotheses, and damage patterns, given the observations from the system response [11, 12]. Thus, the goal of the Bayesian inverse problem proposed herein is threefold: (1) to investigate the posterior PDF of model parameters $\boldsymbol{\theta}$ over the set $\boldsymbol{\Theta} \subset \mathbb{R}^{n_{\boldsymbol{\theta}}}$ of possible values within the model class $\mathcal{M}_{j}$ given the data $\mathcal{D}$, namely $p\left(\boldsymbol{\theta} \mid \mathcal{D}, \mathcal{M}_{j}\right) ;(2)$ to obtain the plausibility of the $j$-th damage hypothesis $\mathcal{M}_{j}$ within the set $\mathbf{M}_{i}=\left\{\mathcal{M}_{1}, \ldots, \mathcal{M}_{j}, \ldots, \mathcal{M}_{N_{M}}\right\}$ of candidates, i.e., $P\left(\mathcal{M}_{j} \mid \mathcal{D}, \mathbf{M}_{i}\right)$; and (3) to investigate the plausibility $P\left(\mathbf{M}_{i} \mid \mathcal{D}, \mathfrak{M}\right)$ of the assumed damage pattern 
$\mathbf{M}_{i}$ within a predefined set $\mathfrak{M}$ of $N_{s}$ plausible candidates, i.e., $\mathfrak{M}=\left\{\mathbf{M}_{1}, \ldots, \mathbf{M}_{i}, \ldots, \mathbf{M}_{N_{S}}\right\}$. Throughout this work, we use $P(\cdot)$ to denote probability whereas a PDF is expressed as $p(\cdot)$. Figure 3 provides a synoptical scheme of the proposed multilevel Bayesian assessment framework.

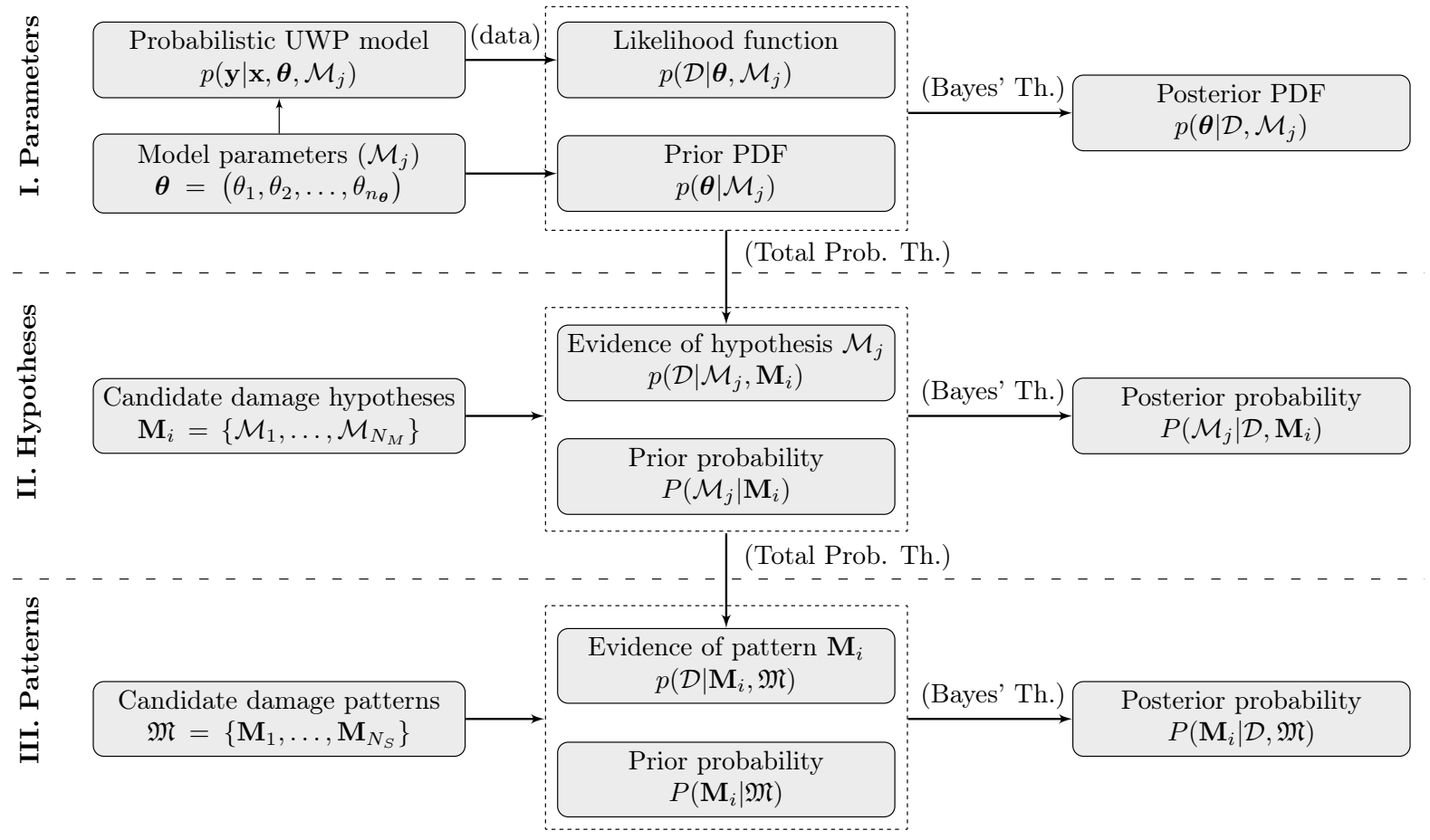

Figure 3: Proposed Bayesian IP framework at the three hierarchical levels considered: (1) Model parameters, (2) damage hypotheses, and (3) damage patterns. Observe that the evidence of the upper levels (II and III) stems from the evidence and the prior probability of the lower levels (I and II, respectively).

\subsubsection{Level 1: Model parameters}

At the level of model parameters, Bayes' Theorem yields the posterior PDF of the parameter vector $\boldsymbol{\theta}$ for a particular model class $\mathcal{M}_{j}$, as follows:

$$
p\left(\boldsymbol{\theta} \mid \mathcal{D}, \mathcal{M}_{j}\right)=c^{-1} p\left(\mathcal{D} \mid \boldsymbol{\theta}, \mathcal{M}_{j}\right) p\left(\boldsymbol{\theta} \mid \mathcal{M}_{j}\right)
$$

where $c$ is a normalizing constant defined so that:

$$
\int_{\Theta} p\left(\boldsymbol{\theta} \mid \mathcal{D}, \mathcal{M}_{j}\right) d \boldsymbol{\theta}=c^{-1} \int_{\Theta} p\left(\mathcal{D} \mid \boldsymbol{\theta}, \mathcal{M}_{j}\right) p\left(\boldsymbol{\theta} \mid \mathcal{M}_{j}\right) d \boldsymbol{\theta}=1
$$

Note that the initial quantification of the plausibility of each model parameterization specified by $\boldsymbol{\theta}$ in $\mathcal{M}_{j}$, which is expressed by the prior $\operatorname{PDF} p\left(\boldsymbol{\theta} \mid \mathcal{M}_{j}\right)$, is updated to obtain the posterior $\operatorname{PDF} p\left(\boldsymbol{\theta} \mid \mathcal{D}, \mathcal{M}_{j}\right)$ by using the information about the system output expressed through the likelihood function $p\left(\mathcal{D} \mid \boldsymbol{\theta}, \mathcal{M}_{j}\right)$. The 


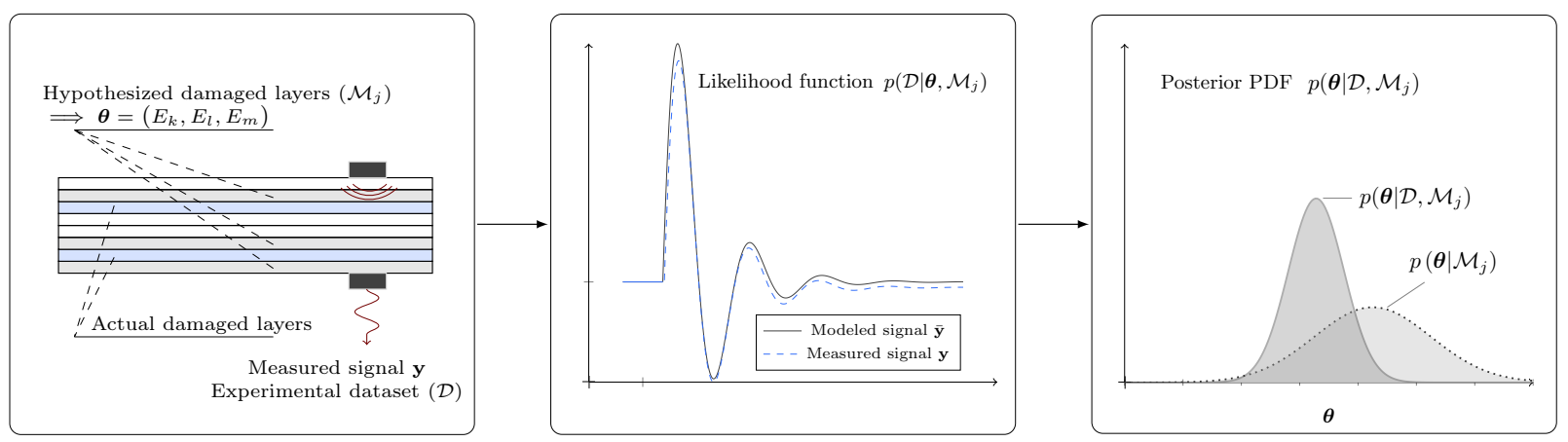

Figure 4: Illustrative example of the Bayesian updating of model parameters for a particular damage hypothesis $\mathcal{M}_{j}$. Left: Schematic section view of a composite laminate with indication of the actual and hypothesized damaged layers, and the model parameterization based on the hypothesized damaged layers. Center: Conceptual illustration of the formulation of the likelihood function (refer also to Figure 2). Right: Final outcome of the model parameter updating through Bayes' Theorem.

likelihood function provides a measure about how likely the ultrasonic data $\mathcal{D}$ are reproduced if the model specified by $\boldsymbol{\theta}$ within model class $\mathcal{M}_{j}$ is adopted. It can be obtained by evaluating the data $\mathcal{D}$ as the outcome of the stochastic UWP model defined in Equation (5). If, instead of a single signal, the data $\mathcal{D}$ consist of a sequence of $N$ ultrasonic signals $\mathcal{D}=\left\{\mathbf{y}_{1}, \ldots, \mathbf{y}_{N}\right\}$ covering a certain damaged area, then the likelihood function can be expressed as:

$$
p\left(\mathcal{D} \mid \boldsymbol{\theta}, \mathcal{M}_{j}\right)=\prod_{n=1}^{N} \underbrace{p\left(\mathbf{y}_{n} \mid \mathbf{x}, \boldsymbol{\theta}, \mathcal{M}_{j}\right)}_{\text {Eq. (5) }}
$$

Figure 4 conceptually illustrates the main steps for Bayesian updating of model parameters for the ultrasoundbased damage identification problem investigated here. It should be noted that one of the main difficulties when applying Bayes' Theorem is that the normalizing constant $c$ cannot be evaluated analytically nor is readily calculated by numerical integration methods, except when the dimension $n_{\boldsymbol{\theta}}$ is small [35]. To tackle this problem, Markov Chain Monte Carlo (MCMC) methods [36, 37] are widely used since they allow obtaining samples from the posterior PDF while circumventing the evaluation of the normalizing constant $c$ in Equation (6) [38-40]. Among them, the Metropolis-Hastings (M-H) algorithm [41, 42] is used here for its versatility and implementation simplicity. The reader is referred to Appendix B for a pseudocode implementation of $\mathrm{M}-\mathrm{H}$ algorithm in the context of this study.

\subsubsection{Level 2: Damage hypotheses}

As stated before, several damage hypotheses may be consistent with the measured signals $\mathcal{D}$. Thus, the goal is to calculate the plausibility of a particular damage hypothesis $\mathcal{M}_{j}$ among the set $\mathbf{M}_{i}=$ $\left\{\mathcal{M}_{1}, \ldots, \mathcal{M}_{j}, \ldots, \mathcal{M}_{N_{M}}\right\}$ of candidates for representing the measured system response. This can be achieved 
by extending Bayes' Theorem at the model class level as follows:

$$
P\left(\mathcal{M}_{j} \mid \mathcal{D}, \mathbf{M}_{i}\right)=\frac{p\left(\mathcal{D} \mid \mathcal{M}_{j}, \mathbf{M}_{i}\right) P\left(\mathcal{M}_{j} \mid \mathbf{M}_{i}\right)}{\sum_{k=1}^{N_{M}} p\left(\mathcal{D} \mid \mathcal{M}_{k}, \mathbf{M}_{i}\right) P\left(\mathcal{M}_{k} \mid \mathbf{M}_{i}\right)}
$$

where $P\left(\mathcal{M}_{j} \mid \mathbf{M}_{i}\right)$ is the prior probability of the $j$-th damage hypothesis in $\mathbf{M}_{i}$, so that $\sum_{j=1}^{N_{M}} P\left(\mathcal{M}_{j} \mid \mathbf{M}_{i}\right)=$ 1. This prior probability expresses the initial modeler's judgement on the relative degree of belief on $\mathcal{M}_{j}$ within the set $\mathbf{M}_{i}$. The factor $p\left(\mathcal{D} \mid \mathcal{M}_{j}, \mathbf{M}_{i}\right)$ is the evidence (also referred to as marginal likelihood) for model class $\mathcal{M}_{j} \in \mathbf{M}_{i}$, and expresses how likely the data $\mathcal{D}$ are reproduced if the damage hypothesis represented by $\mathcal{M}_{j}$ is adopted. The evidence can be calculated using Total Probability Theorem as ${ }^{1}$ :

$$
p\left(\mathcal{D} \mid \mathcal{M}_{j}\right)=\int_{\Theta} p\left(\mathcal{D} \mid \boldsymbol{\theta}, \mathcal{M}_{j}\right) p\left(\boldsymbol{\theta} \mid \mathcal{M}_{j}\right) d \boldsymbol{\theta}
$$

where $p\left(\mathcal{D} \mid \boldsymbol{\theta}, \mathcal{M}_{j}\right)$ and $p\left(\boldsymbol{\theta} \mid \mathcal{M}_{j}\right)$ are the likelihood function and the prior PDF of model parameters, respectively. Once the evidences for the different model classes are obtained, their values allow us ranking the damage hypotheses according to the posterior probabilities given by Equation (9). However, the evaluation of the multi-dimensional integral in Equation (10) is nontrivial except for some particular cases [43], further extended by Yuen and $\mathrm{Mu}$ [44]. In this work, a recent technique based on samples from the posterior PDF $p\left(\boldsymbol{\theta} \mid \mathcal{D}, \mathcal{M}_{j}\right)$ is adopted to numerically solve this integral [45]. More details about this method can be found in [46] in the context of the M-H algorithm used herein.

Note that, in addition to calculate the evidence of a particular damage hypothesis, it is also of much interest to quantify the balance between the data fit in relation to the complexity of such damage hypothesis, i.e., the amount of information extracted from the data. This allows avoiding the over-fitting of the data when the model is unnecessarily adjusted to capture minor details of the ultrasonic signal by means of more complex parameterizations. This can be achieved by examining the evidence of the model class $\mathcal{M}_{j}$ from an information theoretic point of view [11, 26], as follows:

$$
\begin{aligned}
\ln p\left(\mathcal{D} \mid \mathcal{M}_{j}\right) & =\int_{\Theta}\left[\ln p\left(\mathcal{D} \mid \boldsymbol{\theta}, \mathcal{M}_{j}\right)\right] p\left(\boldsymbol{\theta} \mid \mathcal{D}, \mathcal{M}_{j}\right) d \boldsymbol{\theta}-\int_{\boldsymbol{\Theta}}\left[\ln \frac{p\left(\boldsymbol{\theta} \mid \mathcal{D}, \mathcal{M}_{j}\right)}{p\left(\boldsymbol{\theta} \mid \mathcal{M}_{j}\right)}\right] p\left(\boldsymbol{\theta} \mid \mathcal{D}, \mathcal{M}_{j}\right) d \boldsymbol{\theta} \\
& =\mathbb{E}\left[\ln p\left(\mathcal{D} \mid \boldsymbol{\theta}, \mathcal{M}_{j}\right)\right]-\mathbb{E}\left[\ln \frac{p\left(\boldsymbol{\theta} \mid \mathcal{D}, \mathcal{M}_{j}\right)}{p\left(\boldsymbol{\theta} \mid \mathcal{M}_{j}\right)}\right]
\end{aligned}
$$

where $\mathbb{E}$ is the expectation with respect to the posterior $p\left(\boldsymbol{\theta} \mid \mathcal{D}, \mathcal{M}_{j}\right)$. This expression is obtained by strategically multiplying the logarithm of the evidence by a factor of one:

$$
\ln p\left(\mathcal{D} \mid \mathcal{M}_{j}\right)=\ln p\left(\mathcal{D} \mid \mathcal{M}_{j}\right) \underbrace{\int_{\Theta} p\left(\boldsymbol{\theta} \mid \mathcal{D}, \mathcal{M}_{j}\right) d \boldsymbol{\theta}}_{=1}
$$

and then making substitutions according to Bayes' Theorem in Equation (6) to expand the evidence. The first term in Equation (11) accounts for the averaged goodness of fit (AGF) of the model specified by

\footnotetext{
${ }^{1}$ For the sake of clarity, the conditioning on $\mathbf{M}_{i}$ is hereinafter dropped except when necessary.
} 
$\mathcal{M}_{j}$, weighted by the posterior probabilities of model parameters $\boldsymbol{\theta}$. The second term is the relative entropy between the posterior and the prior PDF of model parameters, which determines the Expected Information Gained (EIG) from the data if damage hypothesis represented by $\mathcal{M}_{j}$ is adopted. This term, which is by definition always non-negative, will be larger for more complex damage hypotheses. Therefore, the logevidence of a damage pattern is compounded of a data-fit term and a term that penalizes more complex damage hypotheses, which are those that extract more information from the data to update their prior information. Consequently, Equation (11) allows understanding the correct trade-off between fitting accuracy and model complexity.

\subsubsection{Level 3: Damage patterns}

As explained in Section 2.2, damage hypotheses can be grouped into damage patterns according to the hypothesized number of damaged layers. The posterior plausibility of the candidate damage pattern $\mathbf{M}_{i}$ can be obtained using Bayes' Theorem as follows:

$$
P\left(\mathbf{M}_{i} \mid \mathcal{D}, \mathfrak{M}\right)=\frac{p\left(\mathcal{D} \mid \mathbf{M}_{i}\right) P\left(\mathbf{M}_{i} \mid \mathfrak{M}\right)}{\sum_{j=1}^{N_{S}} p\left(\mathcal{D} \mid \mathbf{M}_{j}\right) P\left(\mathbf{M}_{j} \mid \mathfrak{M}\right)}
$$

where $P\left(\mathbf{M}_{i} \mid \mathfrak{M}\right)$ is the prior plausibility of pattern $\mathbf{M}_{i}$ within the set $\mathfrak{M}=\left\{\mathbf{M}_{1}, \ldots, \mathbf{M}_{i}, \ldots, \mathbf{M}_{N_{s}}\right\}$, and $p\left(\mathcal{D} \mid \mathbf{M}_{i}\right)$ is the evidence of $\mathbf{M}_{i} \in \mathfrak{M}$ for the data $\mathcal{D}$, which expresses how likely the data $\mathcal{D}$ are reproduced if damage pattern $\mathbf{M}_{i}$ is assumed. The evidence $p\left(\mathcal{D} \mid \mathbf{M}_{i}\right)$ can be obtained as a generalization of the evidence calculation at the model class level (recall Eq. (10)) using Total Probability Theorem:

$$
p\left(\mathcal{D} \mid \mathbf{M}_{i}\right)=\sum_{j=1}^{N_{M}} \underbrace{p\left(\mathcal{D} \mid \mathcal{M}_{j}, \mathbf{M}_{i}\right)}_{\text {Eq. (10) }} P\left(\mathcal{M}_{j} \mid \mathbf{M}_{i}\right)
$$

where $P\left(\mathcal{M}_{j} \mid \mathbf{M}_{i}\right)$ is the prior probability of damage hypothesis $\mathcal{M}_{j} \in \mathbf{M}_{i}$, as defined in Section 3.2.2. Observe that, by definition, the number of damage patterns $N_{s} \leqslant N_{L}$ since subscript $i=1, \ldots, N_{S}$ is identified with the dimension of the model parameter space $n_{\boldsymbol{\theta}}$ (recall Section 2.2), which is always less than or equal to the number of layers within the laminate $N_{L}$.

\subsection{Algorithmic implementation}

In a strict sense, a given UWP model parameterized by $\boldsymbol{\theta} \in \mathbb{R}^{n_{\boldsymbol{\theta}}}$ would lead to a set of $N_{M}=\frac{N_{L} !}{\left(N_{L}-n_{\boldsymbol{\theta}}\right) ! n_{\boldsymbol{\theta}} !}$ potential damage hypotheses to be tested using the multilevel Bayesian IP approach presented before. For instance, a laminate with $N_{L}=31$ layers (such as the one considered in the case study below) would lead to $N_{M}=169,911$ potential damage hypotheses to be studied under a specific damage pattern based on $n_{\boldsymbol{\theta}}=5$ damaged layers, which is hardly affordable using standard computational resources. To overcome this drawback, a redefinition of the damage patterns is proposed based on a pre-selection of damage hypotheses as 
follows: ${ }^{2}$ First, an initial damage pattern is defined by considering $n_{\boldsymbol{\theta}}=1$ damaged layers, leading to a set of $N_{M}=N_{L}$ damage hypotheses parameterized by $\boldsymbol{\theta}_{j}^{(1)}=\left(E_{k}\right)$, where $k \in \Xi=\left\{1,2, \ldots, N_{L}\right\}$; henceforth $\mathbf{M}_{1}=\left\{\mathcal{M}_{1}^{(1)}, \ldots, \mathcal{M}_{N_{L}}^{(1)}\right\}$. By Bayesian model class selection (recall Section 3.2.2), the most probable hypothesis $\mathcal{M}_{j^{*}}^{(1)}$ within the set $\mathbf{M}_{1}$ is selected, which is described through a model parameterization specified by $\boldsymbol{\theta}_{j^{*}}^{(1)}=\left(E_{k^{*}}\right)$, where $k^{*} \in \Xi$ is the most probable position of the damaged layer considering damage pattern $\mathbf{M}_{1}$. Next, a second damage pattern based on two damaged layers $\left(n_{\boldsymbol{\theta}}=2\right)$ is defined such that it accepts $N_{L}-1$ damage hypotheses described through the following model parameterization: $\boldsymbol{\theta}_{j}^{(2)}=\left(\boldsymbol{\theta}_{j^{*}}^{(1)}, E_{k}\right)$, which results from augmenting vector $\boldsymbol{\theta}_{j^{*}}^{(1)}$ with parameter $E_{k}$, i.e., $\boldsymbol{\theta}_{j}^{(2)}=\left(E_{k^{*}}, E_{k}\right), k \neq k^{*} \in \Xi$, thus leading to the set $\mathbf{M}_{2}=\left\{\mathcal{M}_{1}^{(2)}, \ldots, \mathcal{M}_{N_{L}-1}^{(2)}\right\}$. Again, the most-probable hypothesis $\mathcal{M}_{j^{*}}^{(2)} \in \mathbf{M}_{2}$ can be selected through Bayesian model class selection, so that $\mathcal{M}_{j^{*}}^{(2)}$ is parameterized by $\boldsymbol{\theta}_{j^{*}}^{(2)}=\left(E_{k^{*}}, E_{l^{*}}\right)$, where $\left\{k^{*}, l^{*}\right\} \in \Xi$ are the most probable positions of the damaged layers under damage pattern $\mathbf{M}_{2}$. The same process is repeated until $N_{S}=N_{L}$ damage patterns have been defined, so that the $i$-th damage pattern within the set $\mathfrak{M}=\left\{\mathbf{M}_{1}, \ldots, \mathbf{M}_{i}, \ldots, \mathbf{M}_{N_{L}}\right\}$ is composed of $N_{M}=N_{L}-i+1$ damage hypotheses parameterized by $\boldsymbol{\theta}_{j}^{(i)}=\left(\boldsymbol{\theta}_{j^{*}}^{(i-1)}, E_{k}\right)=\left(E_{k^{*}}, E_{l^{*}}, \ldots, E_{m^{*}}, E_{k}\right)$, where $k \in \Xi \neq\left\{k^{*}, l^{*}, \ldots, m^{*}\right\}$. A pseudocode of the proposed method is given below as Algorithm 1.

\footnotetext{
${ }^{2}$ For the sake of clarity, subscript $j$ and superscript $(i)$ are employed here to denote the $j$-th damage hypothesis and the $i$-th damage pattern; i.e., $\boldsymbol{\theta}_{j}^{(i)}$ is the model parameter vector for damage hypothesis $\mathcal{M}_{j}$ under damage pattern $\mathbf{M}_{i}$.
} 


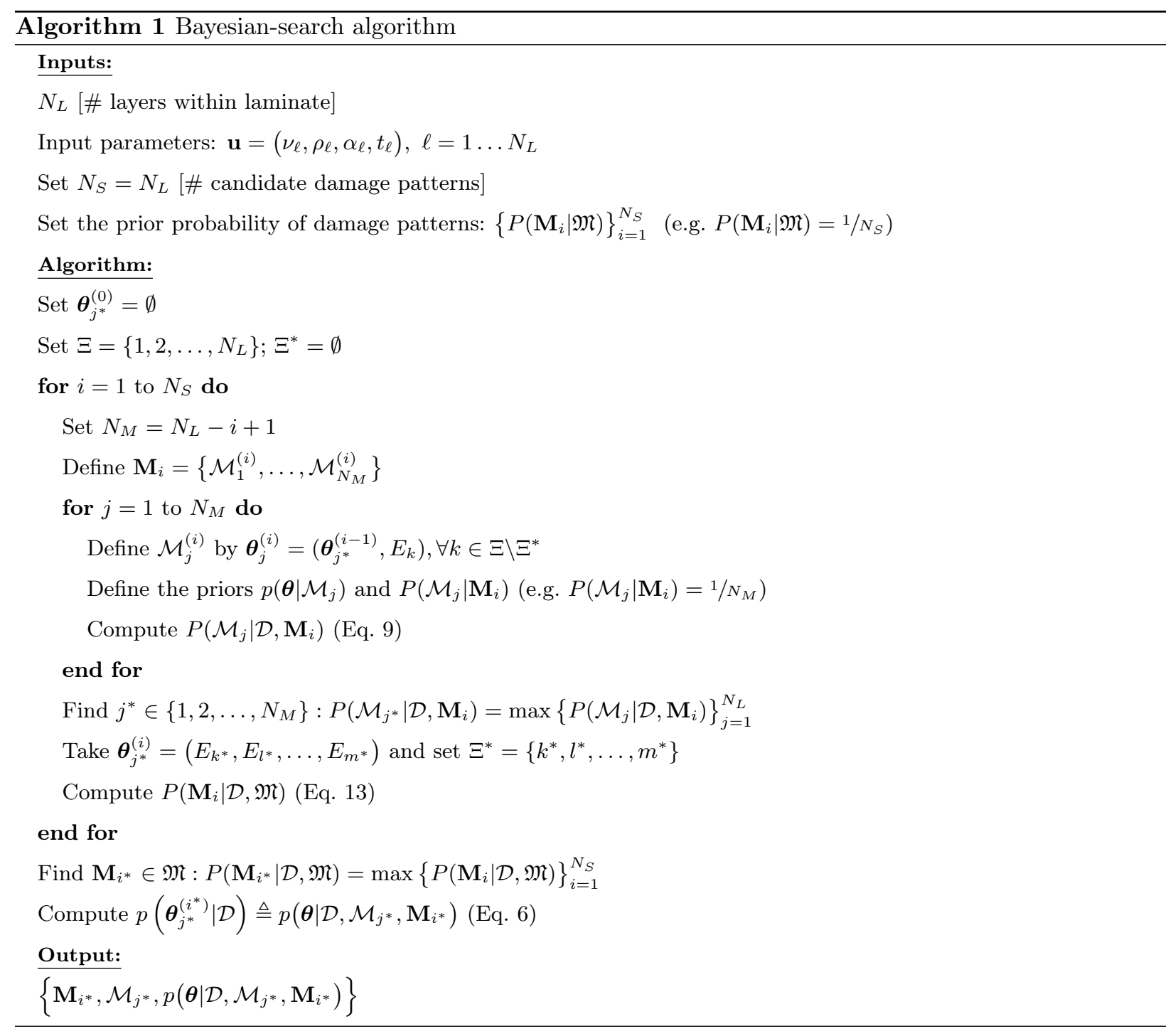

\section{Case studies}

The Bayesian framework for damage identification presented above is exemplified herein using ultrasonic signals from a damaged composite laminate. Section 4.1 describes the experimental setup whereby ultrasonic measurements are acquired. The algorithm settings for Bayesian model updating are presented in Section 4.2. Finally, Section 4.3 introduces the results obtained for Bayesian damage identification.

\subsection{Experimental setup}

The tested material is a cross-ply carbon-epoxy laminate with stacking sequence $[0 / 90]_{4 s}$ exposed to post-impact fatigue damage. Basic mechanical and geometrical properties of this laminate are listed in Table 1. A 3.8 Joule impact damage was introduced in the specimen using a drop weight tower and then 
the laminate was subjected to 100,000 tension-tension fatigue cycles, with a maximum applied load of 20 $\mathrm{KN}$, a frequency $f=6 \mathrm{~Hz}$, and a stress ratio $R=0.1$ (relation between the minimum and maximum stress for each cycle). The damaged specimen was removed from the servo-hydraulic fatigue testing machine and further scanned within an immersion tank (C-scan mode).

\begin{tabular}{lccccc}
\hline Layer properties & $\begin{array}{c}\text { Young's modulus } \\
\bar{E}[\mathrm{GPa}]\end{array}$ & $\begin{array}{c}\text { Poisson ratio } \\
\nu[-]\end{array}$ & $\begin{array}{c}\text { Density } \\
\rho\left[\mathrm{kg} / \mathrm{m}^{3}\right]\end{array}$ & $\begin{array}{c}\text { Attenuation } \\
\alpha[\mathrm{Np} / \mathrm{m}]\end{array}$ & $\begin{array}{c}\text { Thickness } \\
t[\mathrm{~mm}]\end{array}$ \\
\hline Plies $\left(0^{\circ} / 90^{\circ}\right)$ & 11.16 & 0.30 & 1589.5 & 293.02 & 0.12 \\
Interfaces & 5.27 & 0.35 & 1310 & 361.16 & 0.01 \\
\hline
\end{tabular}

Table 1: Nominal values of mechanical and geometrical properties of the intact plies and interfaces of the CFRP $[0 / 90]_{4 s}$ laminate used for the case studies.

For the ultrasonic testing, a through-transmission sub-wavelength technique was adopted whereby longitudinal waves were transmitted normally through the layers, whose thickness is less than or comparable to the wavelength of the emitted wave. Specifically, the damaged specimen was excited by an ultrasonic sine-burst at a central frequency of $5 \mathrm{MHz}$ consisting of one cycle of $0.2 \mu$ s and $5 \mathrm{Vpp}$ amplitude. The specimen was located at the focal distance of the transducers, and scanned over a two-dimensional plane parallel to the transducer areas. Response signals were registered during $10 \mu \mathrm{s}$ and sampled with a high resolution A/D converter after $40 \mathrm{~dB}$ pre-amplification stage, applying a sampling frequency $F_{s}=200 \mathrm{MHz}$ providing $n_{\mathbf{y}}=2000$ samples, which were uniformly quantized with 12 bits. Figure 5 depicts the experimental setup whereby ultrasonic signals were obtained.

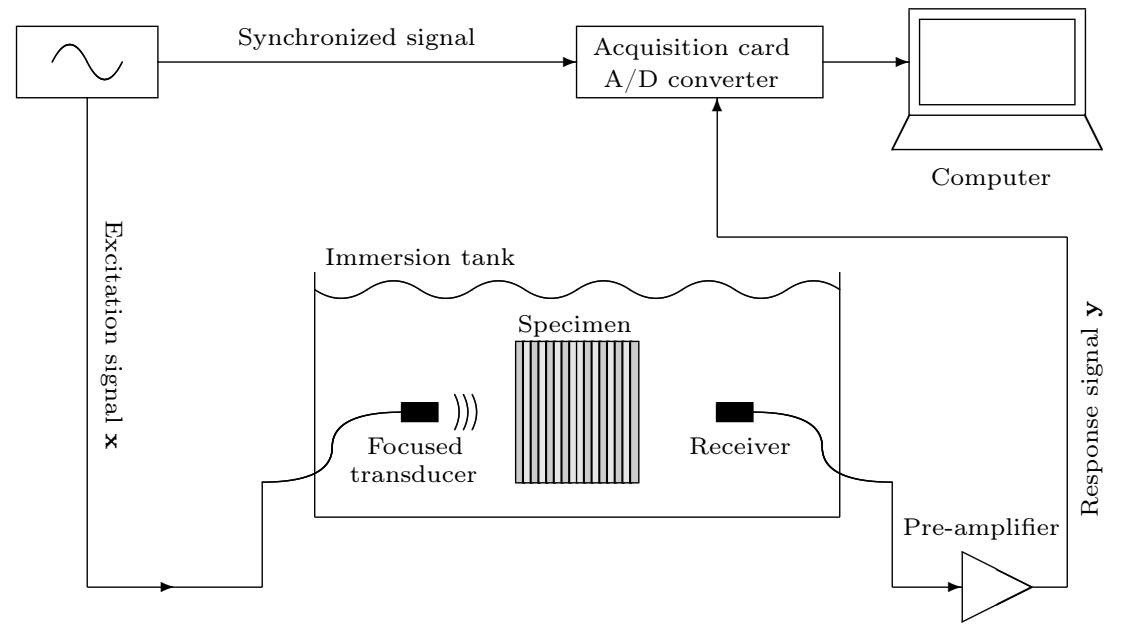

Figure 5: Experimental configuration of the excitation-propagation-measurement system.

Initially, for calibration purposes, the response signal was obtained at an undamaged location (far from the impacted area) and then, the measurement procedure was repeated over an area of $40 \times 20 \mathrm{~mm}^{2}$ centered on the impact location. Three response signals with increasing level of complexity were finally selected. Each 
of these measurements corresponds to the resulting average of 500 captures of the signal, increasing the signal-to-noise ratio around $27 \mathrm{~dB}$.

\subsection{Settings for Bayesian assessment}

Since a major damage mode in composites is delamination, an interface layer of much smaller thickness than both plies and wavelength of the emitted wave is assumed between every two consecutive plies in the digital modeling of the specimen. Thus, the laminate considered in this study is composed by 16 plies plus 15 interfaces, leading to $N_{L}=31$ layers. As explained in Section 2.2, the actual values for Young's moduli of the damaged plies and interfaces were chosen as uncertain model parameters $\boldsymbol{\theta}=\left(E_{k}, E_{l}, \ldots, E_{m}\right)$, which, together with the mechanical properties listed in Table 1, define the input of the UWP model (see Figure 1). Given that the model parameters $\boldsymbol{\theta}$ are always non-negative, their associated prior information was modeled as a lognormal distribution centered at the nominal value of such parameters, i.e., $p\left(E_{\ell} \mid \mathcal{M}\right)=$ $\mathcal{L N}\left(\ln \bar{E}_{\ell}, \sigma_{E_{\ell}}\right)$, being $\bar{E}_{\ell}$ the nominal value of Young's modulus of the $\ell$-th layer (see Table 1 ), and $\sigma_{E_{\ell}}$ the shape parameter of the lognormal distribution. For the case studies investigated in this paper, the selected value for the shape parameter was $\sigma_{E_{\ell}}=0.4 \ln \bar{E}_{\ell}$ for all layers. It should be noted that model parameters were assumed to be stochastically independent, thus the prior PDF of parameters $p(\boldsymbol{\theta} \mid \mathcal{M})$ was defined as the unconditional product of the individual priors, i.e., $p(\boldsymbol{\theta} \mid \mathcal{M})=\prod_{i=1}^{n_{\boldsymbol{\theta}}} p\left(E_{i} \mid \mathcal{M}\right)$. Note that this assumption is not an assertion that no correlations actually exist between model parameters, but a description of the available prior information about such correlations. If they exist, they would become apparent after Bayesian updating [47]. At the model class level, a uniform distribution was adopted for the prior probabilities of damage hypotheses, i.e., $P\left(\mathcal{M}_{j} \mid \mathbf{M}_{i}\right)=1 / N_{M}, j=1, \ldots, N_{M}$. The same probability model was assumed for the prior information of damage patterns, i.e., $P\left(\mathbf{M}_{i} \mid \mathfrak{M}\right)=1 / N_{S}$. Observe from Equations $(9)$ and (13) that computing the evidences of both damage hypotheses and damage patterns is sufficient to assess and rank such hypotheses and patterns because of the aforementioned adoption of equal prior probabilities.

For model parameter updating, the Metropolis-Hastings (M-H) algorithm was applied with a multivariate Gaussian for the proposal PDF, i.e. $q\left(\boldsymbol{\theta}^{\prime} \mid \boldsymbol{\theta}^{\zeta}\right)=\mathcal{N}\left(\boldsymbol{\theta}^{\zeta}, \boldsymbol{\Sigma}_{q}\right)$, where $\boldsymbol{\Sigma}_{q} \in \mathbb{R}^{n_{\boldsymbol{\theta}} \times n_{\boldsymbol{\theta}}}$ is the covariance matrix of the random walk. Further details about M-H implementation are given in Appendix B. Since model parameters $\boldsymbol{\theta}$ were assumed as stochastically independent, $\boldsymbol{\Sigma}_{q}$ is a diagonal matrix, i.e., $\boldsymbol{\Sigma}_{q}=\operatorname{diag}\left(\sigma_{q, 1}^{2}, \ldots, \sigma_{q, n_{\boldsymbol{\theta}}}^{2}\right)$, and each component parameter in $\boldsymbol{\theta}$ performs an independent random walk. The diagonal elements of $\boldsymbol{\Sigma}_{q}$ were appropriately selected through initial test runs such that the monitored acceptance rate (ratio between accepted M-H samples over total amount of samples) is within the suggested range [0.2,0.4] for M-H algorithm [48]. For the definition of the likelihood function (refer to Eq. (5)), the standard deviation of the error parameter is set to $\sigma_{e}=0.075$. This parameter has been shown to have a relatively low influence on the performance of the Bayesian search algorithm, although it could be ideally updated as an additional model parameter within the Bayesian inverse problem. 


\subsection{Results}

\subsubsection{Validation using synthetic signals}

In order to validate the accuracy and robustness of the damage identification performed by the proposed methodology, ground truth data were used consisting of a set of simulated response signals that were directly generated using the UWP model presented in Section 2.1. Damage was artificially introduced in a virtual CFRP $[0 / 90]_{4 s}$ laminate nominally identical to the experimental one by assigning reduced values for the Young's modulus to some layers and interfaces. Three damage scenarios were reproduced such that they were in reasonable agreement with the damage mechanisms that may be expected after a post-impact fatigue damage event $[49,50]$. The assigned values for model parameters in each of the three damage scenarios are listed in Table 2.

\begin{tabular}{llcccccc}
\hline \multirow{2}{*}{ Damage scenario I } & Model parameters $\boldsymbol{\theta}$ & $E_{\ell=1}$ & $E_{\ell=2}$ & $E_{\ell=30}$ & & \\
\cline { 2 - 7 } & Assigned value [GPa] & 8 & 4 & 4 & & \\
\hline \multirow{2}{*}{ Damage scenario II } & Model parameters $\boldsymbol{\theta}$ & $E_{\ell=19}$ & $E_{\ell=29}$ & $E_{\ell=2}$ & $E_{\ell=30}$ & $E_{\ell=18}$ \\
\cline { 2 - 7 } & Assigned value [GPa] & 6 & 9 & 3 & 3.7 & 3.5 \\
\hline \multirow{2}{*}{ Damage scenario III } & Model parameters $\boldsymbol{\theta}$ & $E_{\ell=1}$ & $E_{\ell=17}$ & $E_{\ell=15}$ & $E_{\ell=30}$ \\
\cline { 2 - 7 } & Assigned value [GPa] & 6 & 8 & 8 & 3 \\
\hline
\end{tabular}

Table 2: Assigned values for model parameters used for generating synthetical ultrasound signals considering three damage scenarios for a virtual CFRP $[0 / 90]_{4 s}$ laminate. Subscript $\ell$ denotes the position of the damaged layers defining each damage scenario.

From the assigned values for model parameters, a discrete-time transfer function $H(z ; \boldsymbol{\theta})$ was obtained through Equation (2) for each damage scenario. Consequently, given an excitation signal $\mathbf{x}$, the model for wave propagation enabled us to obtain a simulated response signal $\overline{\mathbf{y}}$ directly by digital filtering. Note that the excitation signal $\mathbf{x}$ used here was a shifted-ahead version of the response signal measured in water (easily obtained by removing the specimen from the immersion tank), since such strategy allows us taking into account the effect of the transducers in the wave propagation simulation. The simulated response signals obtained with the reduced values of Young's modulus were now chosen as the experimental ones to validate the proposed methodology for damage assessment. The goal is twofold: (1) to assess the accuracy of the method in identifying the most probable damage pattern, damage hypothesis, and the most probable values for model parameters among the candidates, and (2) to evaluate the robustness of the identification to signal noise by adding two different levels of white Gaussian noise to the response signals $\overline{\mathbf{y}}$, which respectively correspond to a signal-to-noise ratio (SNR) of 25 and $20 \mathrm{~dB}$.

Table 3 lists the mean and standard deviation of the estimated posterior of model parameters corresponding to the most probable damage hypothesis $\mathcal{M}_{j^{*}}$ within the most evident damage pattern $\mathbf{M}_{i^{*}}$, i.e., $p\left(\boldsymbol{\theta} \mid \mathcal{D}, \mathcal{M}_{j^{*}}, \mathbf{M}_{i^{*}}\right)$. As can be observed, the mean and standard deviation of the estimated Young's moduli are in good agreement with the assigned values for Young's modulus in plies and interfaces. In addition, the 
estimated values for model parameters seem to be rather insensitive to the signal noise level, at least for the SNR considered in this study.

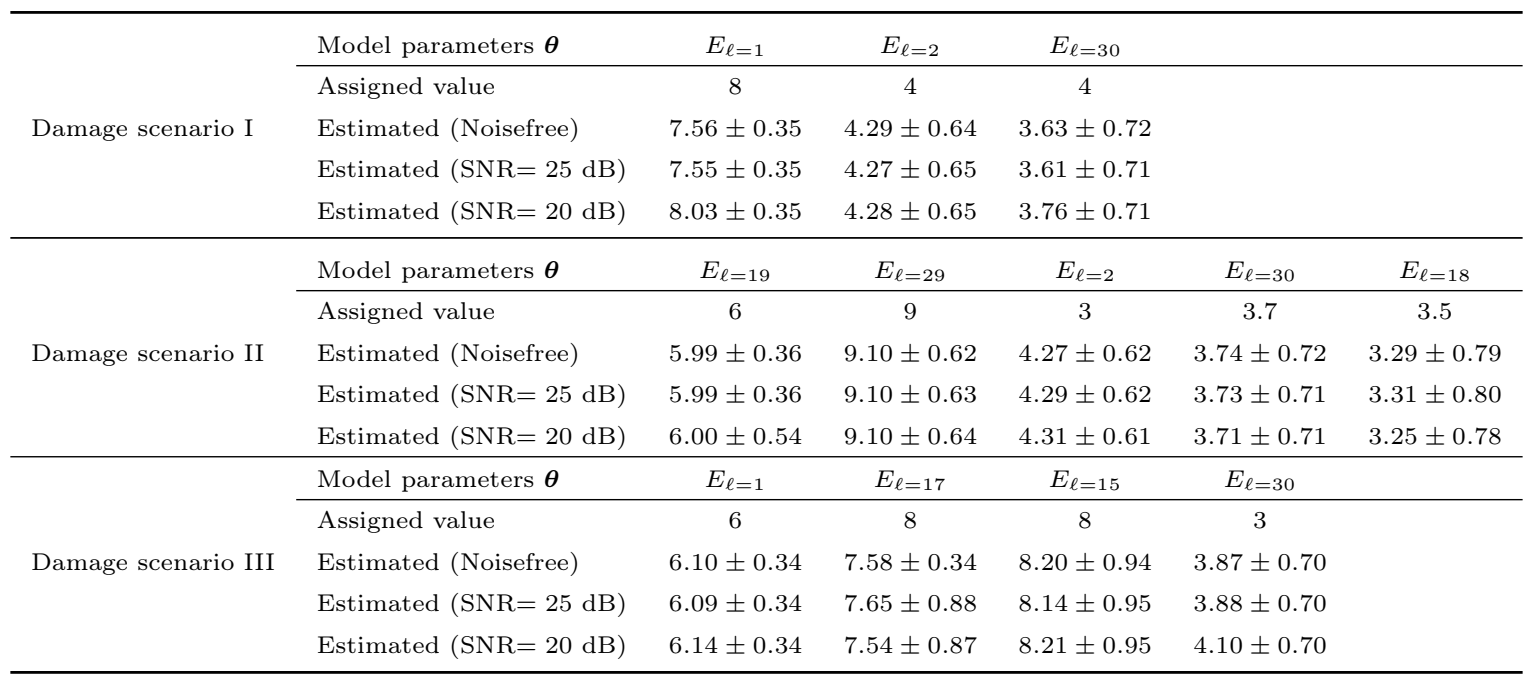

Table 3: Posterior results (mean \pm std) for model parameters considering three different damage scenarios. Results are shown for the damaged layers within the laminate. Odd $\ell$ subscripts are to denote the plies, whilst even $\ell$ subscripts correspond to the interfaces. Units are expressed in GPa.

In Table 4, the through-the-thickness position $\left\{k^{*}, l^{*}, \ldots, m^{*}\right\}$ of the damaged layers as identified by the algorithm is shown for a representative set of seven damage patterns within the set $\mathfrak{M}=\left\{\mathbf{M}_{1}, \mathbf{M}_{2}, \ldots, \mathbf{M}_{31}\right\}$. The identified damaged layers correspond to the most-probable damage hypothesis $\mathcal{M}_{j^{*}}$ for each of the patterns analyzed. The log-evidences of the damage patterns $\ln p\left(\mathcal{D} \mid \mathbf{M}_{i}\right)$ are also listed and the most evident one $\mathbf{M}_{i^{*}} \in \mathfrak{M}$ is marked with bold font. In view of the results, the identification of the damaged layers for the most evident pattern $\mathbf{M}_{i^{*}}$ is consistent with the actual damaged plies selected for signal representation (listed in Table 2), although it seems to fail in the consideration of some interfaces, apparently those placed in the vicinity of previously identified damage plies (e.g., $E_{18}$ in scenario II, and $E_{2}$ in scenario III). This apparent limitation is further analyzed and discussed in Section 5.

As a proof of fitting accuracy, both synthetically generated (experimental) signals $\mathbf{y}$ and modeled signals $\overline{\mathbf{y}}$ are depicted in Figure 6 . The modeled signals $\overline{\mathbf{y}}$ were obtained by averaging the signals obtained by simulating the UWP model using parameter samples from the posterior PDF $p\left(\boldsymbol{\theta} \mid \mathcal{D}, \mathcal{M}_{j^{*}}, \mathbf{M}_{i^{*}}\right)$. Observe that the agreement is particularly remarkable, even in situation where echoes overlap or when echoes are strongly attenuated and drown by noise.

\subsubsection{Evaluation using experimental signals}

In this section, results are provided for Bayesian damage identification using experimental signals obtained from the damaged CFRP $[0 / 90]_{4 s}$ plate described in Section 4.1. The goal is to illustrate the perfor- 


\begin{tabular}{|c|c|c|c|c|c|c|c|c|c|}
\hline & & & $\mathbf{M}_{1}$ & $\mathbf{M}_{2}$ & $\mathbf{M}_{3}$ & $\mathbf{M}_{4}$ & $\mathbf{M}_{5}$ & $\mathbf{M}_{6}$ & $\mathbf{M}_{7}$ \\
\hline \multirow{6}{*}{ Damage scenario I } & \multirow{2}{*}{ Noisefree } & $\mathcal{M}_{j^{*}}^{(i)}$ & $\left(E_{1}\right)$ & $\& E_{2}$ & $\& E_{30}$ & $\& E_{28}$ & $\& E_{26}$ & $\& E_{24}$ & $\& E_{6}$ \\
\hline & & $\ln p\left(\mathcal{D} \mid \mathbf{M}_{i}\right)$ & -5.248 & -1.656 & -1.365 & -1.521 & -2.019 & -2.412 & -2.808 \\
\hline & \multirow{2}{*}{$\mathrm{SNR}=25 \mathrm{~dB}$} & $\mathcal{M}_{j^{*}}^{(i)}$ & $\left(E_{1}\right)$ & $\& E_{2}$ & $\& E_{30}$ & $\& E_{8}$ & $\& E_{6}$ & $\& E_{18}$ & $\& E_{20}$ \\
\hline & & $\ln p\left(\mathcal{D} \mid \mathbf{M}_{i}\right)$ & -6.507 & -2.938 & -2.628 & -2.756 & -3.265 & -3.551 & -4.025 \\
\hline & \multirow{2}{*}{$\mathrm{SNR}=20 \mathrm{~dB}$} & $\mathcal{M}_{j^{*}}^{(i)}$ & $\left(E_{1}\right)$ & $\& E_{2}$ & $\& E_{30}$ & $\& E_{6}$ & $\& E_{24}$ & $\& E_{26}$ & $\& E_{20}$ \\
\hline & & $\ln p\left(\mathcal{D} \mid \mathbf{M}_{i}\right)$ & -9.479 & -6.248 & -6.076 & -6.206 & -6.655 & -7.008 & -7.432 \\
\hline \multirow{6}{*}{ Damage scenario II } & \multirow{2}{*}{ Noisefree } & $\mathcal{M}_{j^{*}}^{(i)}$ & $\left(E_{19}\right)$ & $\& E_{29}$ & $\& E_{2}$ & $\& E_{30}$ & $\& E_{18}$ & $\& E_{14}$ & $\& E_{12}$ \\
\hline & & $\ln p\left(\mathcal{D} \mid \mathbf{M}_{i}\right)$ & -8.900 & -2.983 & -4.687 & -2.133 & -2.179 & -2.364 & -2.853 \\
\hline & \multirow{2}{*}{$\mathrm{SNR}=25 \mathrm{~dB}$} & $\mathcal{M}_{j^{*}}^{(i)}$ & $\left(E_{19}\right)$ & $\& E_{29}$ & $\& E_{2}$ & $\& E_{30}$ & $\& E_{18}$ & $\& E_{14}$ & $\& E_{24}$ \\
\hline & & $\ln p\left(\mathcal{D} \mid \mathbf{M}_{i}\right)$ & -10.582 & -4.692 & -6.402 & -3.837 & -3.875 & -4.112 & -4.585 \\
\hline & \multirow{2}{*}{$\mathrm{SNR}=20 \mathrm{~dB}$} & $\mathcal{M}_{j^{*}}^{(i)}$ & $\left(E_{19}\right)$ & $\& E_{29}$ & $\& E_{2}$ & $\& E_{30}$ & $\& E_{18}$ & $\& E_{26}$ & $\& E_{24}$ \\
\hline & & $\ln p\left(\mathcal{D} \mid \mathbf{M}_{i}\right)$ & -14.300 & -8.437 & -10.154 & -7.557 & -7.590 & -7.887 & -8.449 \\
\hline \multirow{6}{*}{ Damage scenario III } & \multirow{2}{*}{ Noisefree } & $\mathcal{M}_{j^{*}}^{(i)}$ & $\left(E_{1}\right)$ & $\& E_{17}$ & $\& E_{15}$ & $\& E_{30}$ & $\& E_{2}$ & $\& E_{18}$ & $\& E_{6}$ \\
\hline & & $\ln p\left(\mathcal{D} \mid \mathbf{M}_{i}\right)$ & -14.594 & -6.215 & -3.113 & -3.589 & -2.199 & -2.276 & -2.621 \\
\hline & \multirow{2}{*}{$\mathrm{SNR}=25 \mathrm{~dB}$} & $\mathcal{M}_{j^{*}}^{(i)}$ & $\left(E_{1}\right)$ & $\& E_{17}$ & $\& E_{15}$ & $\& E_{30}$ & $\& E_{2}$ & $\& E_{31}$ & $\& E_{18}$ \\
\hline & & $\ln p\left(\mathcal{D} \mid \mathbf{M}_{i}\right)$ & -15.650 & -7.274 & -4.231 & -4.719 & -3.277 & -3.361 & -4.482 \\
\hline & \multirow{2}{*}{$\mathrm{SNR}=20 \mathrm{~dB}$} & $\mathcal{M}_{j^{*}}^{(i)}$ & $\left(E_{1}\right)$ & $\& E_{17}$ & $\& E_{15}$ & $\& E_{30}$ & $\& E_{2}$ & $\& E_{20}$ & $\& E_{28}$ \\
\hline & & $\ln p\left(\mathcal{D} \mid \mathbf{M}_{i}\right)$ & -17.842 & -9.487 & -6.364 & -6.926 & -5.481 & -5.576 & -5.886 \\
\hline
\end{tabular}

Table 4: Most evident damage hypothesis $\mathcal{M}_{j^{*}}^{(i)}$ identified by the algorithm for damage pattern $\mathbf{M}_{i}, i=1, \ldots, 7$, along with their log-evidences $\ln p\left(\mathcal{D} \mid \mathbf{M}_{i}\right)$. The most-evident damage patterns for each of the damage scenarios are marked in bold font. Simplified notation \& $E_{m}$ under pattern $\mathbf{M}_{i}$ denotes that $\mathcal{M}_{j^{*}}^{(i)}$ is given by $\boldsymbol{\theta}_{j^{*}}^{(i)}=\left(\boldsymbol{\theta}_{j^{*}}^{(i-1)}, E_{m}\right)$, where $\boldsymbol{\theta}_{j^{*}}^{(i-1)}=\left(E_{k}, \ldots, E_{l}\right)$ represents the most evident hypothesis within pattern $\mathbf{M}_{i-1}$. For example, \& $E_{28}$ under $\mathbf{M}_{4}$ (see the second row) denotes $\mathcal{M}_{j^{*}}^{(4)}=$ $\left\{E_{1}, E_{2}, E_{30}, E_{28}\right\}$.

mance of the proposed Bayesian methodology in the context of a real-life damaged laminate with a complex post-impact fatigue damage state.

Table 5 shows the posterior mean value and standard deviation of the Young's modulus for the identified damaged layers, which correspond to the most probable damage hypothesis $\mathcal{M}_{j^{*}}$ within the most evident damage pattern $\mathbf{M}_{i^{*}}$. Results are given for three different damage levels (namely, low, moderate, and severe) with increasing complexity in terms of signal attenuation, echoes overlap, and noise. In Table 6, the logevidences of the various damage patterns investigated by the algorithm are shown for the three damage levels considered. It should be noted that, although there is no ground truth data available to validate this experimental case study, results seem consistent with the damage distribution that may be expected in post-impacted fatigue damaged laminates. In particular, as can be observed in Table 5 for the low damage case, the algorithm identifies the first ply (impacted face) and the penultimate $90^{\circ}$-oriented ply as most probable damaged layers, along with the first interface. This result is in accordance with the observations in the open literature about the effects of a low-energy impact on the fatigue damage behavior of CFRP laminates [50-53]. A similar consideration can be drawn for the case of moderate damage, in which, in addition to the external layers, several internal layers within the half part opposite to the impacted face are 

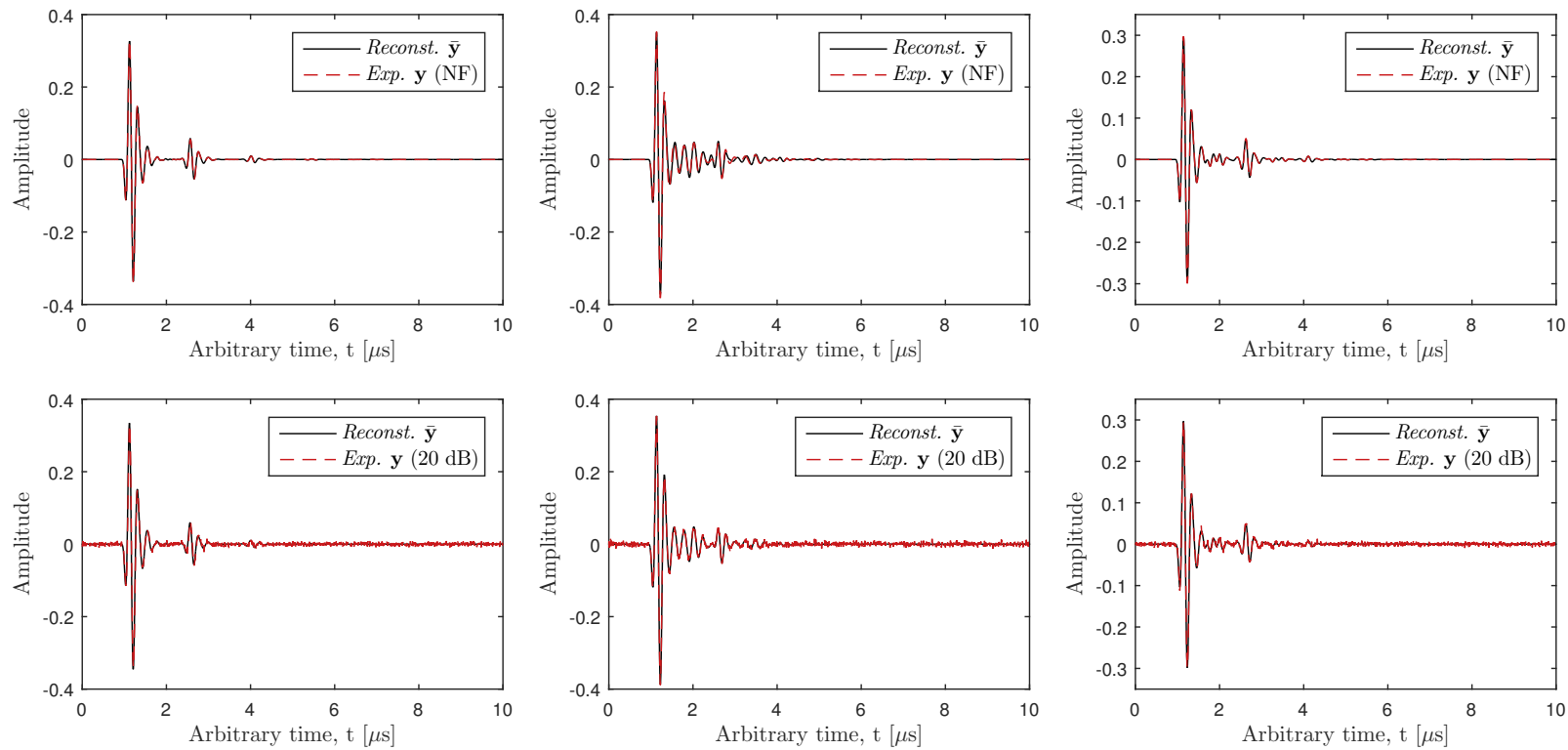

Figure 6: Comparison between the experimental signals (dashed red lines) and the reconstructed signals (solid dark lines) estimated with the proposed Bayesian framework. According to columns, the figures represent damage scenarios I to III from left to right, whereas in rows, the figures represent the noise-free (NF) cases and those with the largest noise level (SNR $=20$ $\mathrm{dB})$.

also identified as most-probable damaged layers.

\begin{tabular}{lccccccccc}
\hline & $\theta_{1}$ & $\theta_{2}$ & $\theta_{3}$ & $\theta_{4}$ & $\theta_{5}$ & $\theta_{6}$ & $\theta_{7}$ & $\theta_{8}$ & $\theta_{9}$ \\
\hline \multirow{2}{*}{ Low } & $E_{1}$ & $E_{2}$ & $E_{29}$ & & & & & & \\
& $10.08 \pm 0.64$ & $6.27 \pm 0.31$ & $3.29 \pm 0.68$ & & & & & & \\
\hline \multirow{2}{*}{ Moderate } & $E_{20}$ & $E_{1}$ & $E_{29}$ & $E_{6}$ & $E_{23}$ & $E_{17}$ & $E_{24}$ & & \\
& $4.22 \pm 0.69$ & $8.29 \pm 0.82$ & $9.73 \pm 1.08$ & $0.64 \pm 0.11$ & $1.68 \pm 0.12$ & $6.34 \pm 0.47$ & $0.67 \pm 0.15$ & & \\
\hline \multirow{2}{*}{ Severe } & $E_{26}$ & $E_{5}$ & $E_{29}$ & $E_{27}$ & $E_{14}$ & $E_{7}$ & $E_{13}$ & $E_{9}$ & $E_{10}$ \\
& $4.22 \pm 0.67$ & $8.35 \pm 1.33$ & $7.56 \pm 1.36$ & $6.56 \pm 1.16$ & $3.14 \pm 0.84$ & $5.46 \pm 0.71$ & $5.56 \pm 0.84$ & $4.70 \pm 1.12$ & $0.06 \pm 0.01$ \\
\hline
\end{tabular}

Table 5: Posterior mean and standard deviation of model parameters corresponding to the most probable hypothesis within the most evident pattern, as depicted in Table 6. Results are shown for the three damage levels considered (low, moderate, severe). Units are expressed in GPa.

Finally, the fit between the experimental signals $\mathbf{y}$ and the signals $\overline{\mathbf{y}}$ obtained by averaging the UWP model using samples from the posterior $\operatorname{PDF} p\left(\boldsymbol{\theta} \mid \mathcal{D}, \mathcal{M}_{j^{*}}, \mathbf{M}_{i^{*}}\right)$ is shown in Figure 7. As can be observed, the agreement between the measured and modeled signals is reasonably good for the cases of low and moderate damage, and sensibly inferior for the case of severe damage. Therefore, the fit to reality may be only partial for this last case, where the noise level is particularly high. 


\begin{tabular}{lccccccccc}
\hline & $\ln p\left(\mathcal{D} \mid \mathbf{M}_{1}\right)$ & $\ln p\left(\mathcal{D} \mid \mathbf{M}_{2}\right)$ & $\ln p\left(\mathcal{D} \mid \mathbf{M}_{3}\right)$ & $\ln p\left(\mathcal{D} \mid \mathbf{M}_{4}\right)$ & $\ln p\left(\mathcal{D} \mid \mathbf{M}_{5}\right)$ & $\ln p\left(\mathcal{D} \mid \mathbf{M}_{6}\right)$ & $\ln p\left(\mathcal{D} \mid \mathbf{M}_{7}\right)$ & $\ln p\left(\mathcal{D} \mid \mathbf{M}_{8}\right)$ & $\ln p\left(\mathcal{D} \mid \mathbf{M}_{9}\right)$ \\
\hline Low & -11.033 & -6.389 & $-\mathbf{6 . 2 5 4}$ & -8.511 & -6.951 & -7.610 & -8.294 & -10.76039 & -9.785 \\
Moderate & -59.708 & -24.866 & -19.611 & -23.388 & -36.284 & -21.965 & $-\mathbf{1 5 . 2 4 6}$ & -19.334 & -20.553 \\
Severe & -67.355 & -14.407 & -13.330 & -11.657 & -11.605 & -16.805 & -12.347 & -11.149 & $-\mathbf{9 . 3 6 0}$ \\
\hline
\end{tabular}

Table 6: Log-evidences of several damage patterns investigated using experimental signals. Three cases are analyzed (low, moderate, severe) corresponding to increasing damage levels. Observe through the highest log-evidence values (in bold font) that the algorithm identifies 3 damaged layers in the low damage case, 7 in the moderate case, and 9 in the severe case. The through-the-thickness distribution of these layers is given in Table 5.
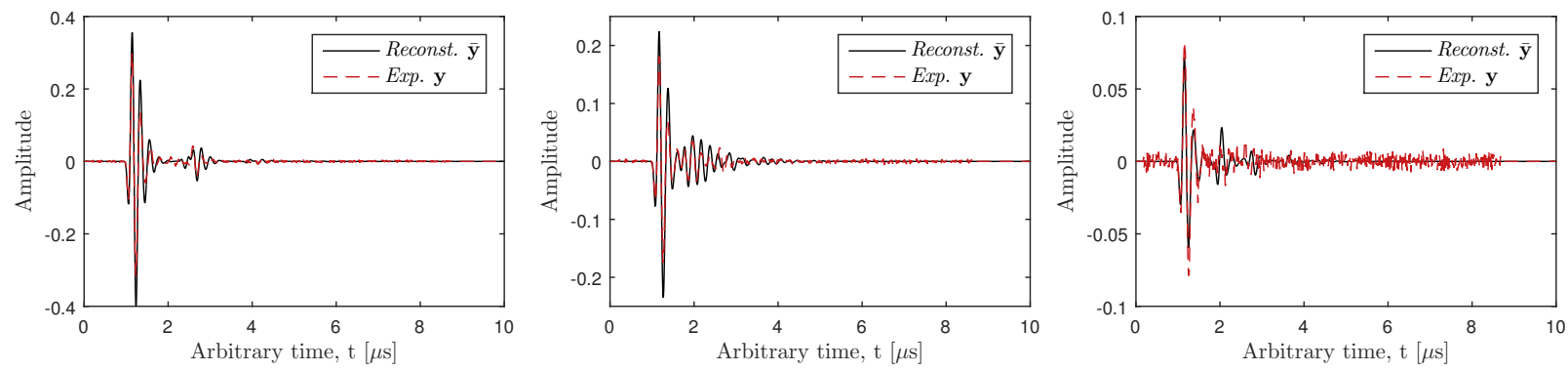

Figure 7: Comparison between the experimental signals (dashed red lines) and the reconstructed signals (solid dark lines). The panels are arranged by columns to represent the three damage levels investigated: Low damage (left), moderate damage (center), and severe damage (right).

\section{Discussion}

The proposed Bayesian methodology for damage assessment has been exemplified using the case studies presented in the previous section. A wave propagation model was used to account for the multiple internal transmissions and reflections following a linear plane wave propagation equation for layered materials. Both synthetically generated and experimentally measured ultrasonic signals were used as experimental data to illustrate the method. As apparent from the results using synthetic signals, the estimated mean values of the Young's modulus converge to the right (assigned) values of model parameters independently of the signal noise, at least for a SNR up to $20 \mathrm{~dB}$ (refer to Table 4). The agreement tends to be better for model parameters corresponding to plies (odd $\ell$-th subscripts in $E_{\ell}$ ), than that corresponding to interfaces (even $\ell$-th subscripts in $E_{i}$ ). An apparent reason is that, at the considered excitation frequency, the model output is more sensitive to a variation of the Young's modulus in the plies, more than to a variation of the Young's modulus in the interfaces, just because plies are about ten times thicker than interfaces.

The last observation is consistent with the results shown in Table 7, where the log-evidences of the most-evident damage hypotheses $\mathcal{M}_{j^{*}}^{(i)}$ are further analyzed using the information-theoretic approach given by Equation (11). In view of the results, one can observe the improvement that can be achieved in the averaged goodness of fit (AGF term) when the Young's modulus parameter of any ply is incorporated 
into the parameterization. However, from a Bayesian perspective, the last also implies a larger information extracted from the ultrasonic data by the UWP model (second term in Eq. (11)), because the high-probability content of the likelihood function is concentrated over the region of these "sensitive" parameters [47], thereby penalizing the evidence of the candidate hypothesis. As observed in Table 7, this balance between the average goodness of fit and the information gained from the data renders the value of the evidence of the overall hypothesis, therefore resulting in a key element for the Bayesian hypothesis assessment.

It should be noted that other methods like the referred AIC and BIC criteria [28, 29] could be considered for the proposed problem of hypothesis selection, as mentioned in Section 1. For discussion purposes, these methods are briefly described below under a uniform notation:

$$
\begin{gathered}
A I C\left(\mathcal{M}_{j} \mid \mathcal{D}\right)=\ln p\left(\mathcal{D} \mid \boldsymbol{\theta}^{*}, \mathcal{M}_{j}\right)-n_{\boldsymbol{\theta}} \\
B I C\left(\mathcal{M}_{j} \mid \mathcal{D}\right)=\ln p\left(\mathcal{D} \mid \boldsymbol{\theta}^{*}, \mathcal{M}_{j}\right)-\frac{1}{2} n_{\boldsymbol{\theta}} \ln n_{\mathbf{y}}
\end{gathered}
$$

where $\boldsymbol{\theta}^{*}$ represents the maximum likelihood value of $\boldsymbol{\theta}$ given model hypothesis denoted by $\mathcal{M}_{j}$. Observe that these methods provide expressions to explicitly perform a trade-off between data-fit and model complexity. However, in view of Equations (15) and (16), the penalty term is based on the number of uncertain parameters $n_{\boldsymbol{\theta}}$, whilst, as shown in Equation (11), the penalty term can greatly differ for two hypotheses even when they share the same $n_{\boldsymbol{\theta}}$, thus biasing the identification $[11,26]$. The last makes the AIC and BIC criteria unsuitable for our problem of hypotheses selection. Moreover, such criteria do not take into account the whole uncertainty of the model parameters, but only a point estimate based on the maximum likelihood value $\boldsymbol{\theta}^{*}$, which may lead to unreliable results for general non-identifiable cases [54]. In contrast, using the Bayesian approach proposed, the whole modeling uncertainty (in both, model parameters and model hypotheses) is considered, and penalties against unnecessary complex models arise in a natural and principled way, as evident from Equation (11). Henceforth, there is no need to introduce any ad-hoc penalty term as with the aforementioned alternative methods.

Besides, observe also that when an interfacial parameter is incorporated into the parameterization, the amount of information extracted from the data is relatively low, since, as stated before, interfacial Young's moduli are low sensitivity parameters. Nevertheless, depending on the particular case, the addition of a new interfacial parameter has been shown to provide slight improvements in the averaged data-fit of the candidate damage hypothesis, usually when such parameter corresponds to an interface in the vicinity of a previously identified damaged ply. The last may lead to a biased damage hypothesis selection when the difference between the prior and posterior PDFs of these added interfacial parameters is relatively small (e.g., $10 \%$ stiffness decrease), since this small change for a low sensitivity parameter implies a very low penalty term in Equation (11), and thus, a trend to use these parameters to overfit the signal. However, it should be noted that damage at interfaces is usually due to delamination damage, which actually leads to a severe stiffness reduction. Therefore, the aforementioned bias may vanish by forcing larger values of 
degradation for these parameters through a suitable redefinition of their prior PDFs.

\begin{tabular}{|c|c|c|c|c|}
\hline & Damage hypothesis & $\ln p\left(\mathcal{D} \mid \mathcal{M}_{j^{*}}, \mathbf{M}_{i}\right)$ & AGF & EIG \\
\hline \multirow{4}{*}{ Damage scenario I (20 dB) } & $\mathcal{M}_{j^{*}}^{(1)}:\left\{E_{1}\right\}$ & -6.4423 & -5.3676 & 1.0747 \\
\hline & $\mathcal{M}_{j^{*}}^{(2)}:\left\{E_{1}, E_{2}\right\}$ & -5.8326 & -5.0981 & 0.7344 \\
\hline & $\mathcal{M}_{j^{*}}^{(3)}:\left\{E_{1}, E_{2}, E_{30}\right\}$ & -5.8309 & -5.0534 & 0.7774 \\
\hline & $\mathcal{M}_{j^{*}}^{(4)}:\left\{E_{1}, E_{2}, E_{30}, E_{6}\right\}$ & -6.0454 & -5.1536 & 0.8918 \\
\hline \multirow{5}{*}{ Damage scenario II (20 dB) } & $\mathcal{M}_{j^{*}}^{(1)}:\left\{E_{19}\right\}$ & -10.8667 & -7.8509 & 3.0158 \\
\hline & $\mathcal{M}_{j^{*}}^{(2)}:\left\{E_{19}, E_{29}\right\}$ & -7.66010 & -6.7773 & 0.8828 \\
\hline & $\mathcal{M}_{j^{*}}^{(3)}:\left\{E_{19}, E_{29}, E_{2}\right\}$ & -9.3616 & -6.4508 & 2.1081 \\
\hline & $\mathcal{M}_{j^{*}}^{(4)}:\left\{E_{19}, E_{29}, E_{2}, E_{30}\right\}$ & -7.2077 & -6.1171 & 1.0906 \\
\hline & $\mathcal{M}_{j^{*}}^{(5)}:\left\{E_{19}, E_{29}, E_{2}, E_{30}, E_{18}\right\}$ & -7.3337 & -6.1270 & 1.2067 \\
\hline \multirow{5}{*}{ Damage scenario III (20 dB) } & $\mathcal{M}_{j^{*}}^{(1)}:\left\{E_{1}\right\}$ & -14.4105 & -10.5483 & 3.8622 \\
\hline & $\mathcal{M}_{j^{*}}^{(2)}:\left\{E_{1}, E_{17}\right\}$ & -7.3765 & -5.6256 & 1.7508 \\
\hline & $\mathcal{M}_{j^{*}}^{(3)}:\left\{E_{1}, E_{17}, E_{15}\right\}$ & -5.7937 & -4.3861 & 1.4076 \\
\hline & $\mathcal{M}_{j^{*}}^{(4)}:\left\{E_{1}, E_{17}, E_{15}, E_{30}\right\}$ & -6.5557 & -4.1705 & 2.3852 \\
\hline & $\mathcal{M}_{j^{*}}^{(5)}:\left\{E_{1}, E_{17}, E_{15}, E_{30}, E_{2}\right\}$ & -5.2451 & -3.9588 & 1.2863 \\
\hline
\end{tabular}

Table 7: Information-theoretic approach to the evidence of most probable damage hypothesis $\mathcal{M}_{j *}^{(i)}$ within the $i$-th damage pattern considered (AGF = Average Goodness of Fit, EIG = Expected Information Gain). Results are shown for three different damage scenarios. The values shown for the log-evidence $\ln p\left(\mathcal{D} \mid \mathcal{M}_{j}, \mathbf{M}_{i}\right)$ (third column) correspond to the difference from those shown in forth and fifth columns, respectively. Observe that damaged plies (odd values for subscript $\ell$ in $E_{\ell}$ ) are generally identified first.

Another limitation of this research is the simplification adopted in the parameterization of the UWP model based on the Young's modulus of plies and interfaces, which does not account for other physical variables (e.g., attenuation coefficient) as uncertain parameters. However, allowing all model inputs as updatable parameters would yield a highly dimensional inverse problem where the uncertainties on the parameters would cross-propagate creating a bias in the identification [47]. In this context, a desirable further work would be the analysis of the "optimal" model parameterization from an uncertainty quantification point of view, for example by using Global Sensitivity Analysis [55]. Analyzing the effect of this optimal parameterization on the topology of the likelihood function, and thus, on the evidence of the model class, would be also beneficial. An additional further work would be the cross-validation of the proposed Bayesian damage assessment methodology for real damaged laminates using complementary structural health monitoring techniques (e.g., by in-situ dynamic scanning electron microscopy (SEM)).

In summary, the results have highlighted the relevance of the hierarchical Bayesian approach for damage identification proposed in this paper. The complexity of the damage distribution across the layers makes it necessary to reduce the number of uncertain parameters that describe the model, in order to avoid overfitting and gain certainty on the actual values of damage parameters. The amount of information extracted from the data emerges as a key variable for the overall assessment of the candidate hypothesis represented by a particular model parameterization, and, as a consequence, for the assessment of the damage pattern that 
contains such hypothesis. Then, the evidence of the damage hypothesis accounts for such information gain as a penalty term and implicitly enforces a quantitative Ockham's razor, such that simpler damage hypotheses that are consistent with the data are favoured through a healthy balance between the information gained from the data and the average goodness of fit.

\section{Conclusion}

A novel Bayesian approach for damage identification in layered materials is presented based on throughtransmission ultrasonic measurements using a wave propagation model and a multilevel probabilistic reconstruction algorithm. Both synthetically generated and experimentally obtained ultrasonic signals with different levels of noise and complexity were used as experimental signals to illustrate the proposed methodology. The number and location of damaged layers along with the actual value of their Young's modulus were estimated through Bayes' Theorem based on relative probabilities that measure the extent of agreement between the measured signal and the signal obtained by the wave propagation model. A key contribution in the context of ultrasound-based damage assessment is the avoidance of the necessity to adopt a predefined hypothesis on the damage distribution to solve the inverse problem. More research effort is needed to asses an optimal model parameterization for the ultrasound wave propagation model, and in general, to extend and cross-validate this approach using other structural health monitoring techniques.

\section{Acknowledgements}

Authors acknowledge the University of Granada (Spain) for "Contrato-Puente 2014/2015" postdoctoral fellowships and the European Union for project GGI3000IDIB which partially support this research. In addition, authors would like to thank Dr. Henrik Schmutzler and Prof. Karl Schulte from Technical University of Hamburg-Harburg (Germany) for providing us the damaged samples, and finally Prof. James L. Beck from Caltech (USA) for his valuable guidance through Bayesian methodology.

\section{Appendix A. Recursive approach for the digital model}

It can be proven that for the product of $N_{L}$-layers, a $z$-transformed transfer matrix $\boldsymbol{T}(z)$ that relates the input and output displacement fields has the following general form [13]:

$$
\boldsymbol{T}(z)=\left(\prod_{\ell=1}^{N_{L}} \frac{G_{\alpha_{\ell}} z^{-m_{\ell}}}{1+G_{r_{\ell}}}\right)\left(\begin{array}{cc}
P_{N_{L}}\left(G_{\alpha}, z\right) & \left(\prod_{\ell=1}^{N_{L}} G_{\alpha_{\ell}}^{-2} z^{2 m_{\ell}}\right) Q_{N_{L}}\left(G_{\alpha}^{-1}, z^{-1}\right) \\
Q_{N_{L}}\left(G_{\alpha}, z\right) & \left(\prod_{\ell=1}^{N_{L}} G_{\alpha_{\ell}}^{-2} z^{2 m_{\ell}}\right) P_{N_{L}}\left(G_{\alpha}^{-1}, z^{-1}\right)
\end{array}\right)
$$


The functions $P_{N_{L}}\left(G_{\alpha}, z\right)$ and $Q_{N_{L}}\left(G_{\alpha}, z\right)$ stand for polynomials of $\left(N_{L}-1\right)$-order which are built up following a recursive scheme,

$$
\begin{aligned}
& P_{N_{L}}\left(G_{\alpha}, z\right)=P_{N_{L}-1}\left(G_{\alpha}, z\right)+G_{r_{N_{L}}} G_{\alpha_{N_{L}}}^{-2} z^{2 m_{N_{L}}} Q_{N_{L}-1}\left(G_{\alpha}, z\right) \\
& Q_{N_{L}}\left(G_{\alpha}, z\right)=G_{r_{N_{L}}} P_{N_{L}-1}\left(G_{\alpha}, z\right)+G_{\alpha_{N_{L}}}^{-2} z^{2 m_{N_{L}}} Q_{N_{L}-1}\left(G_{\alpha}, z\right)
\end{aligned}
$$

with $P_{1}\left(G_{\alpha}, z\right)=1$ and $Q_{1}\left(G_{\alpha}, z\right)=G_{r_{1}}$. These polynomials incorporate all the multiple transmissions/reflections and attenuation effects of the multilayered structure. Inserting the boundary conditions for a throughtransmission configuration yields the following discrete-time transfer function $H(z)$,

$$
H(z)=\frac{\prod_{\ell=1}^{N_{L}} G_{t_{\ell}} G_{\alpha_{\ell}} z^{-m_{\ell}}}{P_{N_{L}}\left(G_{\alpha}^{-1}, z^{-1}\right)-\left(\prod_{\ell=1}^{N_{L}} G_{\alpha_{\ell}}^{2} z^{-2 m_{\ell}}\right) Q_{N_{L}}\left(G_{\alpha}, z\right)}
$$

A bare inspection of Equation (A.3) reveals that the lowest polynomial coefficient of the denominator is provided by $P_{1}\left(G_{\alpha}^{-1}, z^{-1}\right)=1$, ensuring that the filter is causal and realizable. On the other hand, the highest coefficient is $2 \Lambda$, with $\Lambda=\sum_{\ell=1}^{N_{L}} m_{\ell}$, due to the term $\prod_{\ell=1}^{N_{L}} z^{-2 m_{\ell}}$ in the denominator. Consequently, the denominator can be replaced by $1+\sum_{k=1}^{2 \Lambda} a_{k} z^{-k}$, as shown in Equation (2). Additionally, the numerator $\prod_{\ell=1}^{N_{L}} G_{t_{\ell}} G_{\alpha_{\ell}} z^{-m_{\ell}}$ can be replaced by $b_{0} z^{-\Lambda}$, i.e., a gain corresponding to the multiplication of the transmission $G_{t_{\ell}}$ and attenuation coefficients $G_{\alpha_{\ell}}$ across all layers plus a total thickness sample delay.

\section{Appendix B. Metropolis-Hastings simulation for Bayesian updating}

M-H algorithm generates samples from a specially constructed Markov chain whose stationary distribution is the required posterior $\operatorname{PDF} p(\boldsymbol{\theta} \mid \mathcal{D}, \mathcal{M})$. By sampling a candidate model parameter $\boldsymbol{\theta}^{\prime}$ from a proposal distribution $q\left(\boldsymbol{\theta}^{\prime} \mid \boldsymbol{\theta}^{\zeta}\right)$, the M-H obtains the state of the chain at $\zeta+1$, given the state at $\zeta$, specified by $\boldsymbol{\theta}^{\zeta}$. The candidate parameter $\boldsymbol{\theta}^{\prime}$ is accepted (i.e., $\boldsymbol{\theta}^{\zeta+1}=\boldsymbol{\theta}^{\prime}$ ) with probability $\min \{1, r\}$, and rejected (i.e., $\boldsymbol{\theta}^{\zeta+1}=\boldsymbol{\theta}^{\zeta}$ ) with the remaining probability $1-\min \{1, r\}$, where:

$$
r=\frac{p\left(\mathcal{D} \mid \boldsymbol{\theta}^{\prime}, \mathcal{M}\right) p\left(\boldsymbol{\theta}^{\prime} \mid \mathcal{M}\right) q\left(\boldsymbol{\theta}^{\zeta-1} \mid \boldsymbol{\theta}^{\prime}\right)}{p\left(\mathcal{D} \mid \boldsymbol{\theta}^{\zeta-1}, \mathcal{M}\right) p\left(\boldsymbol{\theta}^{\zeta-1} \mid \mathcal{M}\right) q\left(\boldsymbol{\theta}^{\prime} \mid \boldsymbol{\theta}^{\zeta-1}\right)}
$$

The process is repeated until $T_{s}$ samples have been generated so that the monitored acceptance rate (ratio between accepted M-H samples over total amount of samples) reaches an asymptotic behaviour. A pseudocode description of this method is provided below as Algorithm 2. 


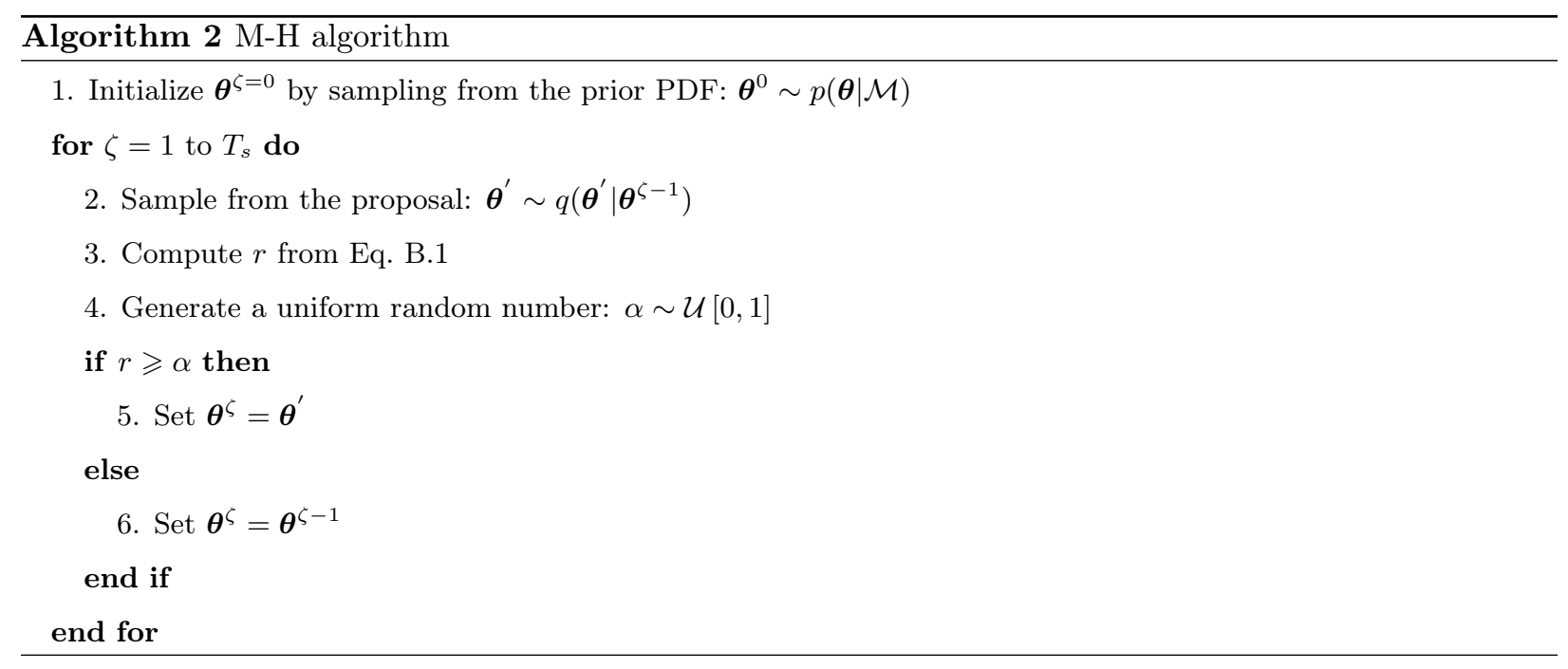

\section{References}

[1] D. Hull, T. Clyne, An Introduction to Composite Materials, Cambridge University Press, 1996.

[2] D.-H. Kim, Composite Structures for Civil and Architectural Engineering, E \& FH Spon, 1995.

[3] R. Jamison, K. Schulte, K. Reifsnider, W. Stinchcomb, Characterization and analysis of damage mechanisms in tensiontension fatigue of graphite/epoxy laminates, Effects of defects in composite materials, ASTM STP 836 (1984) 21-55.

[4] R. Talreja, C. V. Singh, Damage and Failure of Composite Materials, Cambridge University Press, 2012.

[5] K. Reifsnider, A. Talug, Analysis of fatigue damage in composite laminates, International Journal of Fatigue 2 (1) (1980) $3-11$.

[6] S. Wooh, I. Daniel, Enhancement techniques for ultrasonic nondestructive evaluation of composite materials, Journal of Engineering Materials and Technology 112 (2) (1990) 175-182.

[7] A. Fahim, R. Gallego, N. Bochud, G. Rus, Model-based damage reconstruction in composites from ultrasound transmission, Composites Part B: Engineering 45 (1) (2013) 50-62.

[8] M. Bonnet, A. Constantinescu, Inverse problems in elasticity, Inverse Problems 21 (2) (2005) R1-R50.

[9] S. Avril, M. Bonnet, A.-S. Bretelle, M. Grediac, F. Hild, P. Ienny, F. Latourte, D. Lemosse, S. Pagano, E. Pagnacco, et al., Overview of identification methods of mechanical parameters based on full-field measurements, Experimental Mechanics 48 (4) (2008) 381-402.

[10] A. Tarantola, Inverse Problem Theory and Methods for Model Parameters Estimation, SIAM, 2005.

[11] J. L. Beck, Bayesian system identification based on probability logic, Structural Control and Health Monitoring 17 (2010) $825-847$.

[12] G. Rus, J. Chiachío, M. Chiachío, Logical inference for inverse problems, Inverse Problems in Science and Engineering 24 (3) (2016) 448-464.

[13] N. Bochud, A. Gomez, G. Rus, A. Peinado, A sparse digital signal model for ultrasonic nondestructive evaluation of layered materials, Ultrasonics 62 (2015) 160-173.

[14] J. Rowatt, P. Spanos, Markov chain models for life prediction of composite laminates, Structural Safety 20 (1998) $117-135$.

[15] B.-S. Wei, S. Johnson, R. Haj-Ali, A stochastic fatigue damage method for composite materials based on Markov chains and infrared thermography, International Journal of Fatigue 32 (2) (2010) 350-360.

[16] S. Sankararaman, S. Mahadevan, Integration of model verification, validation, and calibration for uncertainty quantification in engineering systems, Reliability Engineering \& System Safety 138 (2015) 194-209. 
[17] A Bayesian statistical inference approach to the non-destructive inspection of composite material, in: X. Gros (Ed.), \{NDT $\}$ Data Fusion, Butterworth-Heinemann, Oxford, 1996, Ch. 5, pp. 95-126.

[18] T. Peng, A. Saxena, K. Goebel, Y. Xiang, S. Sankararaman, Y. Liu, A novel Bayesian imaging method for probabilistic delamination detection of composite materials, Smart Materials and Structures 22 (12) (2013) 125019.

[19] M. Gobbato, J. P. Conte, J. B. Kosmatka, C. R. Farrar, A reliability-based framework for fatigue damage prognosis of composite aircraft structures, Probabilistic Engineering Mechanics 29 (2012) 176-188.

[20] T. Peng, Y. Liu, A. Saxena, K. Goebel, In-situ fatigue life prognosis for composite laminates based on stiffness degradation, Composite Structures 132 (0) (2015) 155-165.

[21] J. Chiachío, M. Chiachío, S. Sankararaman, A. Saxena, K. Goebel, Condition-based prediction of time-dependent reliability in composites, Reliability Engineering \& System Safety 142 (2015) 134-147.

[22] N. Bochud, G. Rus, Probabilistic inverse problem to characterize tissue-equivalent material mechanical properties, IEEE Transactions on Ultrasonics, Ferroelectrics and Frequency Control 59 (7) (2012) 1443-1456.

[23] L. Peralta, G. Rus, N. Bochud, F. Molina, Assessing viscoelasticity of shear wave propagation in cervical tissue by multiscale computational simulation, Journal of Biomechanics 48 (9) (2015) 1549-1556.

[24] S. F. Gull, Bayesian inductive inference and maximum entropy, in: Maximum-entropy and Bayesian methods in science and engineering, Springer, 1988, pp. 53-74.

[25] E. Jaynes, Probability Theory: The Logic of Science, Cambridge University Press, 2003.

[26] M. Muto, J. L. Beck, Bayesian updating and model class selection for hysteretic structural models using stochastic simulation, Journal of Vibration and Control 14 (1-2) (2008) 7-34.

[27] H. Akaike, A new look at the statistical model identification, IEEE Transactions on Automatic Control 19 (6) (1974) $716-723$.

[28] H. Akaike, On entropy maximization principle, Application of Statistics (1976) 27-41.

[29] G. Schwarz, Estimating the dimension of a model, The Annals of Statistics 6 (2) (1978) $461-464$.

[30] J. Proakis, D. Manolakis, Digital Signal Processing, Principles, Algorithms, and Applications, Pentice Hall, 1996.

[31] V. K. Kinra, C. Zhu, Ultrasonic nondestructive evaluation of thin (sub-wavelength) coatings, The Journal of the Acoustical Society of America 93 (5) (1993) 2454-2467.

[32] E. Jaynes, Information theory and statistical mechanics, The Physical Review 106 (4) (1957) 620-630.

[33] R. T. Cox, The Algebra of Probable Inference, The Johns Hopkins University Press, 1961.

[34] E. T. Jaynes, Papers on Probability, Statistics and Statistical physics, (Ed. R.D. Rosenkrantz) Kluwer Academic Publishers, 1983.

[35] J. L. Beck, S.-K. Au, Bayesian updating of structural models and reliability using Markov chain Monte Carlo simulation, Journal of Engineering Mechanics 128 (4) (2002) 380-391.

[36] W. Gilks, S. Richardson, D. J. Spiegelhalter, Markov Chain Monte Carlo in Practice, Chapman and Hall, 1996.

[37] R. Neal, Probabilistic inference using Markov chain Monte Carlo methods, Intelligence 62.

[38] A. F. M. Smith, G. O. Roberts, Bayesian computation via the Gibbs sampler and related Markov chain Monte Carlo methods, Journal of the Royal Statistical Society. Series B (Methodological) 55 (1) (1993) 3-23.

[39] D. Gamerman, H. F. Lopes, Markov chain Monte Carlo: stochastic simulation for Bayesian inference, CRC Press, 2006.

[40] F. Liang, C. Liu, J. Chuanhai, Advanced Markov chain Monte Carlo methods, Wiley Online Library, 2010.

[41] N. Metropolis, A. W. Rosenbluth, M. N. Rosenbluth, A. H. Teller, E. Teller, Equation of state calculations by fast computing machines, The Journal of Chemical Physics 21 (1953) 1087-1092.

[42] W. Hastings, Monte Carlo sampling methods using Markov chains and their applications, Biometrika 57 (1) (1970) 97-109.

[43] J. L. Beck, K.-V. Yuen, Model selection using response measurements: Bayesian probabilistic approach, Journal of Engineering Mechanics 130 (2) (2004) 192-203. 
[44] K.-V. Yuen, H.-Q. Mu, Peak ground acceleration estimation by linear and nonlinear models with reduced order Monte Carlo simulation, Computer-Aided Civil and Infrastructure Engineering 26 (1) (2011) 30-47.

[45] S. H. Cheung, J. L. Beck, Calculation of posterior probabilities for Bayesian model class assessment and averaging from posterior samples based on dynamic system data, Computer-Aided Civil and Infrastructure Engineering 25 (5) (2010) $304-321$

[46] M. Chiachío, J. Chiachío, G. Rus, J. L. Beck, Predicting fatigue damage in composites: A Bayesian framework, Structural Safety 51 (2014) 57-68.

[47] J. Chiachío, M. Chiachío, A. Saxena, S. Sankararaman, G. Rus, K. Goebel, Bayesian model class selection and parameter estimation for fatigue damage progression in composites, International Journal of Fatigue 70 (2015) 361-373.

[48] G. Roberts, J. Rosenthal, Optimal scaling for various Metropolis-Hastings algorithms, Statistical Science 16 (4) (2001) $351-367$.

[49] W. Cantwell, P. Curtis, J. Morton, Impact and subsequent fatigue damage growth in carbon fibre laminates, International Journal of Fatigue 6 (2) (1984) 113-118.

[50] N. Tai, M. Yip, J. Lin, Effects of low-energy impact on the fatigue behavior of carbon/epoxy composites, Composites Science and Technology 58 (1) (1998) 1-8.

[51] F. Aymerich, S. Meili, Ultrasonic evaluation of matrix damage in impacted composite laminates, Composites Part B: Engineering 31 (1) (2000) 1-6.

[52] A. Chen, D. Almond, B. Harris, Impact damage growth in composites under fatigue conditions monitored by acoustography, International Journal of Fatigue 24 (2) (2002) 257-261.

[53] N. Uda, K. Ono, K. Kunoo, Compression fatigue failure of CFRP laminates with impact damage, Composites Science and Technology 69 (14) (2009) 2308-2314.

[54] L. S. Katafygiotis, J. L. Beck, Updating models and their uncertainties. II: Model identifiability, Journal of Engineering Mechanics 124 (4) (1998) 463-467.

[55] A. Saltelli, M. Ratto, T. Andres, F. Campolongo, J. Cariboni, D. Gatelli, M. Saisana, S. Tarantola, Global Sensitivity Analysis: The Primer, Wiley-Interscience, 2008. 